\title{
Initial powers of Sturmian sequences
}

\author{
by \\ ValÉRIe Berthé (Montpellier), Charles Holton (Austin, TX) \\ and Luca Q. Zamboni (Denton, TX)
}

\section{INTRODUCTION}

There are a number of recent papers on powers of words occurring in Sturmian sequences (see for instance $[1,2,3,6,8,9,16,17,27,32,38$, 41]). Quantities of interest include the supremum of powers of factors of a sequence (the index or critical exponent of the sequence), and the limit superior of powers of longer and longer factors of the sequence. It is well known that these numbers are finite if and only if the partial quotients of the continued fraction expansion of the slope of the Sturmian sequence are bounded (see [31]). An explicit formula for the index of a Sturmian sequence was given by Vandeth (see Theorem 16 in [41]) in terms of the partial quotients of its slope.

This paper deals with powers of factors occurring at the beginning of Sturmian sequences, which we call initial powers. The work is motivated in part by a simple observation about the Fibonacci Sturmian shift, the shift space of all Sturmian sequences of slope $2 /(1+\sqrt{5})$. This space is infinite, minimal and uniquely ergodic; one might expect prefix powers to be somewhat uniform. Yet its characteristic sequence begins in no $(3+\sqrt{5}) / 2 \approx 2.62$ power at all, while every sequence outside the shift orbit of the characteristic sequence begins in arbitrarily long words repeated three or more times. This example leads us to define the initial critical exponent of a sequence $\omega$ over a finite alphabet, denoted ice $(\omega)$, as the supremum of all real numbers $p>0$ for which there exist arbitrarily long prefixes $u$ of $\omega$ such that $u^{p}$ is also a prefix of $\omega$. We obtain an explicit formula for the initial critical exponent of a Sturmian sequence, in terms of a particular $S$-adic expansion. For characteristic Sturmian sequences, our formula for ice has probably been known

2000 Mathematics Subject Classification: Primary 37B10; Secondary 37A25, 11J70, $68 \mathrm{R} 15$.

The second author was partially supported by NSF grant DMS-0091946.

The third author was partially supported by NSF grant INT-9726708. 
since [34], though Hedlund and Morse did not address this question specifically. One can also obtain the formula for ice of a characteristic sequence using Cassaigne's formula for the recurrence quotient in [13]. See also [9, 43].

Every Sturmian sequence $\omega$ on the alphabet $\{0,1\}$ admits a unique $S$ adic representation as an infinite composition of the form

$$
\omega=T^{c_{1}} \circ \tau_{0}^{a_{1}} \circ T^{c_{2}} \circ \tau_{1}^{a_{2}} \circ T^{c_{3}} \circ \tau_{0}^{a_{3}} \circ T^{c_{4}} \circ \tau_{1}^{a_{4}} \circ \cdots,
$$

where $T$ denotes the one-sided shift map, $\tau_{0}$ and $\tau_{1}$ are the morphisms on $\{0,1\}^{*}$ defined by

$$
\begin{array}{ll}
\tau_{0}(0)=0, & \tau_{1}(0)=10, \\
\tau_{0}(1)=01, & \tau_{1}(1)=1,
\end{array}
$$

$a_{k} \geq c_{k} \geq 0$ for all $k, a_{k} \geq 1$ for $k \geq 2$, and if $c_{k}=a_{k}$ then $c_{k-1}=0$. The sequence $\left(a_{k}\right)_{k \geq 1}$ turns out to be the sequence of partial quotients of the slope (defined as the density of the symbol 1), while $\left(c_{k}\right)_{k \geq 1}$ is the sequence of digits in the arithmetic Ostrowski expansion of the intercept of the Sturmian sequence (see for instance [18, 19, 28, 29, 26, 35, 39, 40] and the references in [10]). From this point of view, the characteristic (or standard) Sturmian sequence of a particular slope is the one having $c_{k}=0$ for all $k$. This expansion of $\omega$ is just one of many possible expansions as an infinite composition of morphisms (see work of Arnoux [37], ArnouxFisher [5], Arnoux-Ferenczi-Hubert [4]). In each case these expansions are intimately linked to the Ostrowski numeration system.

In [3] it is shown that each Sturmian sequence begins in infinitely many squares (see also [16]), and hence ice $(\omega) \geq 2$ for all Sturmian sequences $\omega$. We show that the value 2 is attainable, and give the following characterization of those slopes for which there is a Sturmian sequence with initial critical exponent equal to 2 :

TheOREM 1.1. Let $\alpha=\left[0 ; a_{1}, a_{2}, a_{3}, \ldots\right]$ be an irrational number and let $X_{\alpha}$ be the set of all Sturmian sequences of slope $\alpha$. Then there is a Sturmian sequence $\omega \in X_{\alpha}$ with ice $(\omega)=2$ if and only if for each pair of positive integers $(s, t)$ with $s>1$ there are only finitely many indices $k$ for which $\left(a_{k}, a_{k+1}\right)=(s, t)$ or $\left(a_{k}, a_{k+1}, a_{k+2}\right)=(1,1, t)$.

We also show how to explicitly construct a Sturmian sequence $\omega \in X_{\alpha}$ with $\operatorname{ice}(\omega)=2$ in case one exists.

Write ind ${ }^{*}(\omega)$ for the limit superior of powers of longer and longer words appearing in a sequence $\omega$. We prove the following relation between ice and ind* for characteristic Sturmian sequences:

TheOREm 1.2. Let $\omega$ be the characteristic Sturmian sequence of slope $\alpha$. Then

$$
\operatorname{ind}^{*}(\alpha)=1+\operatorname{ice}(\omega)
$$


The paper is organized as follows. After first recalling some basic facts on Sturmian sequences and on ice, we introduce in Section 2 two $S$-adic representations of Sturmian sequences (additive and multiplicative versions) based on Ostrowski's numeration system, and conclude the section with a characterization of primitive substitutive Sturmian sequences. We derive an explicit formula for the ice of a Sturmian sequence in Section 3. We study general properties of ice in Section 4; special attention is given to the Fibonacci shift in Section 4.4. We end with a proof of Theorem $1.1 \mathrm{in}$ Section 5 .

\section{PRELIMINARIES}

2.1. Definitions and notation. Throughout the paper, $\alpha$ denotes an irrational number in $(0,1)$. Consider two two-interval exchange transformations, $R_{\alpha}:[-\alpha, 1-\alpha) \rightarrow[-\alpha, 1-\alpha)$ and $\widetilde{R}_{\alpha}:(-\alpha, 1-\alpha] \rightarrow(-\alpha, 1-\alpha]$, defined by

$$
\begin{aligned}
& R_{\alpha}(z)= \begin{cases}z+\alpha & \text { if } z \in[-\alpha, 1-2 \alpha), \\
z+\alpha-1 & \text { if } z \in[1-2 \alpha, 1-\alpha),\end{cases} \\
& \widetilde{R}_{\alpha}(z)= \begin{cases}z+\alpha & \text { if } z \in(-\alpha, 1-2 \alpha], \\
z+\alpha-1 & \text { if } z \in(1-2 \alpha, 1-\alpha] .\end{cases}
\end{aligned}
$$

Both can be considered as rotations of angle $2 \pi \alpha$, since these are conjugate, after identification of points $-\alpha$ and $1-\alpha$, to a circle rotation. A Sturmian sequence $\omega \in\{0,1\}^{\mathbb{N}}$ of slope $\alpha$ is simply the forward itinerary (with respect to the natural partition) of a point $x \in[-\alpha, 1-\alpha]$ (called the intercept of $\omega$ ) under the action of one of these transformations, i.e., either

$$
\forall k \in \mathbb{N}\left(\omega_{k}=0 \Leftrightarrow R_{\alpha}^{k}(x) \in[-\alpha, 1-2 \alpha)\right)
$$

or

$$
\forall k \in \mathbb{N}\left(\omega_{k}=0 \Leftrightarrow \widetilde{R}_{\alpha}^{k}(x) \in(-\alpha, 1-2 \alpha]\right) .
$$

It is clear from this interpretation that the slope of a Sturmian sequence is the density of the symbol 1.

Notation. In all that follows, the coding of the orbit of the point $y$ with respect to the partition $(I, J)$ under the action of the two-interval exchange $E$ means the sequence $v \in\{0,1\}^{\mathbb{N}}$ defined by

$$
\forall k \in \mathbb{N}\left(v_{k}=0 \Leftrightarrow E^{k}(y) \in I\right) .
$$

A factor of a sequence $\omega$ is a finite subsequence of the form $\omega[i, j):=$ $\omega_{i} \omega_{i+1} \ldots \omega_{j-1}$, i.e., a finite word that appears in $\omega$. The complexity function $p: \mathbb{N} \rightarrow \mathbb{N}$ for a sequence $\omega$ is given by

$$
p(n)=\text { the number of distinct factors of } \omega \text { of length } n \text {. }
$$


Sturmian sequences are exactly those one-sided infinite sequences with complexity $p(n)=n+1$ for every $n$ (see $[34,14]$ ). Write $X_{\alpha}$ for the set of all Sturmian sequences of slope $\alpha$, and denote by $T$ the shift map on sequences, i.e., $(T(\omega))_{i}=\omega_{i+1}$. Then $X_{\alpha}$ is a compact, $T$-invariant subset of $\{0,1\}^{\mathbb{N}}$ and the restriction of $T$ to $X_{\alpha}$ (we shall abuse notation and call it $T$ also) gives us an infinite, minimal, uniquely ergodic (one-sided) shift space. Recall that a map on a topological space is minimal if the only closed nonempty invariant subset is the whole space, and is uniquely ergodic if there exists a unique invariant Borel probability measure on the space. The characteristic sequence of slope $\alpha$ is the unique left-special sequence in $X_{\alpha}$, i.e., the sequence having more than one $T$-preimage in $X_{\alpha}$; this is the sequence with intercept 0 (it is the same for $R_{\alpha}$ and $\widetilde{R}_{\alpha}$ ) and its two shift preimages code respectively the orbits of $-\alpha$ under $R_{\alpha}$ and $1-\alpha$ under $\widetilde{R}_{\alpha}$. For more details on Sturmian sequences, see [30, 37].

We shall use in Sections 2.3 and 2.4 the notion of induction of a rotation. The induced transformation of the rotation $R_{\alpha}$ (or similarly of $\widetilde{R}_{\alpha}$ ) on a subinterval $I$ of $[-\alpha, 1-\alpha]$ is defined as follows. For $x \in I$, we call the first return time of $x$ in $I$ and denote by $n_{I}(x)$ the smallest integer $m>0$ such that $R_{\alpha}^{m}(x) \in I$ ( $m$ is finite since $\alpha$ is irrational). The induced transformation of $R_{\alpha}$ on $I$ is the map $x \mapsto R_{\alpha}^{n_{I}(x)}(x)$ on $I$.

A sequence is called recurrent if each of its factors appears infinitely many times, and uniformly recurrent if each of its factors appears with bounded gaps. If a shift space is minimal, then any of its sequences is uniformly recurrent, and any shift space generated by a uniformly recurrent sequence, as the closure of the orbit of this sequence under the action of the shift, is minimal. A shift space $(X, T)$ is said to be linearly recurrent if there exists a constant $K$ such that, for each $n \in \mathbb{N}$, every factor of length $n$ of a sequence of $X$ appears in every factor of length $n K$. If a shift space $(X, T)$ is linearly recurrent, then it is minimal, and it has sublinear complexity, that is, there exists $C>0$ such that, for all $n \in \mathbb{N}$, there are at most $C n$ different factors of length $n$ in sequences of $X$. For more details on these notions, see for instance [37] and [21]. If $i \in\{0,1\}$ we denote by $\bar{\imath}$ the other symbol in $\{0,1\}$. Thus $\bar{\imath}=1-i, \tau_{i}(i)=i$, and $\tau_{i}(\bar{\imath})=i \bar{\imath}$. Throughout the paper we write $\theta$ for the golden mean, $(1+\sqrt{5}) / 2$. We use Greek letters $\omega$ and $v$ for infinite sequences, and Roman letters $u, v, w$ for finite words. The length of a word $w$ over the alphabet $\{0,1\}$ is denoted by $|w|$. We write $\mathbb{N}$ for the set of nonnegative integers $(0 \in \mathbb{N})$ and $\mathbb{N}^{*}$ for the set of positive integers.

2.2. Initial critical exponent. Positive integer powers of a finite word $w$ are defined by

$$
w^{1}=w \quad \text { and } \quad w^{n}=w^{n-1} w \quad \text { for } n>1 .
$$


We define $w^{0}$ to be the empty word, i.e., the unique word of length 0 , and for arbitrary $p \geq 0$, the $p$ th power of $w$ is given by

$$
w^{p}=w^{\lfloor p\rfloor} u
$$

where $u$ is the prefix of $w$ of length $\lceil(p-\lfloor p\rfloor)|w|\rceil$. Thus, $w^{p}$ has length $\lceil p|w|\rceil$. A word is called primitive if it is not an integer power of some shorter word. The power of a word $w$ in a sequence $\omega$ is the largest $p$ (possibly $\infty$ ) so that $w^{p}$ is a factor of $\omega$. The prefix power of a word $w$ in a sequence $\omega$ is the largest $p$ (possibly $\infty$ ) so that $w^{p}$ is a prefix of $\omega$. We define the initial critical exponent of $\omega$, denoted by ice $(\omega)$, as the limit superior of the prefix powers of the words $\omega[0, n)$ in $\omega$. We similarly define $\operatorname{ind}^{*}(\omega)$ for a sequence $\omega$ as the limit superior as $n$ tends to $\infty$ of the largest powers of the factors of length $n$ appearing in $\omega$. For a minimal shift space $X$, we write $\operatorname{ind}^{*}(X)$ for the common value of ind* on sequences of $X$. Let us prove some properties of ice and ind*.

Proposition 2.1. Let $(X, T)$ be a (one-sided) shift space. Then:

(1) For any $\omega \in X$ one has ice $(\omega) \leq \mathrm{ice}(T \omega)$, and if the inequality is strict then $T \omega$ is the shift image of at least two different members of $X$, i.e., $\omega$ is a left-special element of $X$.

(2) If $(X, T)$ is minimal then $\max _{\omega \in X} \operatorname{ice}(\omega)=\operatorname{ind}^{*}(X)$.

(3) If $X$ is infinite and minimal then some $\omega \in X$ has ice $(\omega) \leq 1+\theta=$ $(3+\sqrt{5}) / 2$.

(4) If $(X, T)$ is minimal with sublinear complexity then ice is shift invariant off of the union of a finite set of orbits; hence ice is almost everywhere constant with respect to any ergodic Borel measure.

(5) If $(X, T)$ is linearly recurrent then ice is almost everywhere equal to $\operatorname{ind}^{*}(X)$ with respect to any finite invariant Borel measure.

Proof. Let $\omega \in X$. If $w$ is a prefix of $\omega$ with prefix power $p$ then the first right conjugate of $w$, i.e., the word $v$ obtained from $w$ by moving the first letter to the end, is a prefix of $T \omega$ with prefix power $p-1 /|w|$. The inequality in (1) follows by taking limits as $|w|$ tends to infinity.

Now suppose the inequality in (1) is strict. Then ice $(T \omega)>1$. Let $v_{k}$ be an increasing sequence of prefixes of $T \omega$ whose corresponding prefix powers $q_{k}$ converge to ice $(T \omega)$. Let $a$ be the first letter of $\omega$ and let $b$ be a common last letter for infinitely many of the $v_{k}$. By passing to a subsequence we may assume that $q_{k}>1$ and $v_{k}$ ends in $b$ for all $k$. Note that $a \neq b$, since otherwise, for all $k$, the first left conjugate of $v_{k}$ is a prefix of $\omega$ with prefix power $q_{k}+1 /\left|v_{k}\right|$ and we obtain a contradiction:

$$
\lim _{k \rightarrow \infty} q_{k}+\frac{1}{\left|v_{k}\right|} \leq \operatorname{ice}(\omega)<\operatorname{ice}(T \omega)=\lim _{k \rightarrow \infty} q_{k} .
$$


For each $k, T \omega$ begins in $v_{k} v_{k}^{q_{k}-1}$ and $\omega$ begins in $a v_{k}^{q_{k}}$, hence $a v_{k}^{q_{k}-1}$ and $b v_{k}^{q_{k}-1}$ are both factors of sequences of $X$. But $\left|v_{k}^{q_{k}-1}\right| \rightarrow \infty$ and each $v_{k}^{q_{k}-1}$ is a prefix of $T \omega$, hence $a T \omega$ and $b T \omega$ both belong to $X$.

To prove (2) we need the following:

$\left(2^{\prime}\right)$ For every $p \in\left(0\right.$, ind $\left.^{*}(X)\right)$, every word which appears in sequences of $X$ is a prefix of some word whose $p$ th power appears in sequences of $X$.

Proof of $\left(2^{\prime}\right)$. By minimality, if $w$ appears in sequences of $X$ then it appears in bounded gaps, i.e., there exists $N=N(w)$ such that for all $\omega$ in $X$, at least one of $\omega, T \omega, \ldots, T^{N(w)-1} \omega$ begins in $w$. Choose $\eta>0$ such that $p+\eta<\operatorname{ind}^{*}(X)$, and let $v$ be a word of length at least $N / \eta$ such that $v^{p+\eta}$ appears in sequences of $X$. Then one of the first $N-1$ right conjugates of $v$ has $w$ as a prefix and appears to power $p$ in sequences of $X$.

Proof of (2). By $\left(2^{\prime}\right)$ we can find a sequence $w_{k}$ of words which appear in sequences of $X$, such that, for each $k, w_{k}^{p_{k}}$ is a prefix of $w_{k+1}$, where $p_{k} \geq 1$ and $p_{k} \rightarrow \operatorname{ind}^{*}(X)$ and $\left|w_{k}\right| \rightarrow \infty$ as $k \rightarrow \infty$. There is a unique $\omega \in X$ having each $w_{k}$ as a prefix, and the construction guarantees ice $(\omega) \geq \operatorname{ind}^{*}(X)$. We always have ice $\leq \operatorname{ind}^{*}(X)$, of course.

Part (3) follows from [33].

To prove (4), we use Cassaigne's result from [12]: The first difference of the complexity function is bounded if complexity is sublinear. Let $C>0$ be an upper bound for the first difference of the complexity. By minimality, every word $w$ in $X$ of length $n$ has at least one left extension, that is, a word aw occurring in $X$ for some letter $a$; hence there can be no more than $C$ words of length $n$ which have two or more left extensions, and the set of sequences $\omega$ in $X$ that have more than one shift preimage has at most $C$ elements.

Assertion (5) holds trivially if $X$ consists of a single periodic orbit, so let us assume that $X$ is an infinite set. Then $(X, T)$ is minimal and has sublinear complexity, and $\operatorname{ind}^{*}(X)<\infty$. Let $\mu$ be a (nonzero) finite invariant Borel measure for $(X, T)$. Suppose, for a contradiction, that $\mu\{\omega \in X$ : $\left.\operatorname{ice}(\omega)<\operatorname{ind}^{*}(X)\right\}>0$. For some $\varepsilon>0$ we must have $\mu\{\omega \in X$ : ice $(\omega)<$ $\left.\operatorname{ind}^{*}(X)-\varepsilon\right\}>0$. Fix such an $\varepsilon$, and set

$$
E=\left\{\omega \in X: \operatorname{ice}(\omega)<\operatorname{ind}^{*}(X)-\varepsilon\right\} .
$$

Let $\nu$ be the Borel probability measure defined by

$$
\nu(B)=\frac{\mu(E \cap B)}{\mu E} .
$$

As in the proof of part (4), ice is invariant off a finite set of orbits. Thus, $\mu\left(T^{-1} E \backslash E\right)=0$, and $\nu$ is an invariant measure. Choose a subsequence $\left(n_{k}\right)_{k \geq 0}$ of the positive integers such that the sequence of maximal powers 
$p_{k}$ of words of length $n_{k}$ converges to ind* $(X)$. Linear recurrence implies that the $p_{k}$ are bounded and the $\nu$-measure of the set of sequences beginning in a word of length $n_{k}$ to power at least $p_{k}-\varepsilon$ is bounded away from 0 (see for instance [21]). But then we must have $\nu\left\{\omega \in X: \operatorname{ice}(\omega) \geq \operatorname{ind}^{*}(X)-\varepsilon\right\}>0$, a contradiction.

The focus of this paper is on the values of ice on the set $X_{\alpha}$ of all Sturmian sequences of some fixed irrational slope $\alpha$. It follows from known results (see for instance [41]) that

$$
\operatorname{ind}^{*}(\alpha):=\operatorname{ind}^{*}\left(X_{\alpha}\right)=2+\limsup _{k \rightarrow \infty}\left[a_{k} ; a_{k-1}, \ldots, a_{1}\right],
$$

where $\left[a_{k} ; a_{k-1}, \ldots, a_{1}\right]$ denotes the continued fraction of $a_{k}, a_{k-1}, \ldots, a_{1}$. This implies in particular that any Sturmian sequence contains cubes (see also [9]) and that a Sturmian sequence has finite index if and only if its slope has bounded partial quotients (this last result is in [32]). See [13] for a study of the topological structure of the set of values taken by the index. Recall that $X_{\alpha}$ is uniquely ergodic.

Lemma 2.2. The almost everywhere value of ice on $X_{\alpha}$ is $\operatorname{ind}^{*}(\alpha)$.

Proof. Suppose first that $\operatorname{ind}^{*}(\alpha)=\infty$. Let $p>2$ and $N \geq 3$. There is a primitive word $u$ of length at least $N$ and a power $p^{\prime} \geq N p+1$ such that $u^{p^{\prime}}$ appears in $X_{\alpha}$ and the exponent $p^{\prime}$ is maximal for words having the same length as $u$.

We claim that $u^{p^{\prime}-1}$ is left special, i.e., both $0 u^{p^{\prime}-1}$ and $1 u^{p^{\prime}-1}$ appear in $X_{\alpha}$. To see this, let $a$ be the last letter of $u$. Since $a u^{p^{\prime}}$ is the same as the first left conjugate of $u$ to power $p^{\prime}+1 /|u|$, maximality of $p^{\prime}$ implies that this word does not appear in $X_{\alpha}$. One of the symbols $b \in\{0,1\}$ is such that $b u^{p^{\prime}}$ appears in $X_{\alpha}$, and we have just shown that $b \neq a$. Thus $a u^{p^{\prime}-1}$ and $b u^{p^{\prime}-1}$ both appear in $X_{\alpha}$, the former as a suffix of $u^{p^{\prime}}$ and the latter as a prefix of $b u^{p^{\prime}}$.

A return word to a factor $h$ in a sequence $\omega$ is a factor $\omega[i, j)$, where $h$ occurs in $\omega$ starting at the $i$ th and $j$ th places and nowhere between. Sturmian sequences have the following properties (see for instance [42]):

- there is exactly one left special factor of each length;

- every factor has exactly two return words;

- the sum of the lengths of the return words to a factor $v$ is at least $|v|+1 ;$ and

- the length of a return word to a left special factor $v$ is bounded above by $|v|+1$.

We know that $u$ is a return word for $u^{p^{\prime}-1}$ because $u$ is primitive and $p^{\prime}>3$. The other return word to $u^{p^{\prime}-1}$ must be a prefix of $u^{p^{\prime}-1} 0$ or $u^{p^{\prime}-1} 1$ of length at least $\left(p^{\prime}-2\right)|u|+1$. This implies that the set of points of $X_{\alpha}$ beginning 
in a suffix of $u^{p^{\prime}-1}$ of length at least $\left(p^{\prime}-1\right)|u| / N$ has measure at least

$$
\frac{\left\lceil\frac{(N-1)\left(p^{\prime}-1\right)|u|}{N}\right\rceil}{\left(p^{\prime}-2\right)|u|+1} \geq \frac{N-1}{N}
$$

and such points begin in a word of length $|u|$ to power $p$. The result follows easily from this.

In case $\operatorname{ind}^{*}(\alpha)<\infty$, the partial quotients of $\alpha$ are bounded and $X_{\alpha}$ is linearly recurrent following [21]. Part (5) of Proposition 2.1 applies directly.

Using this lemma and the formula for $\operatorname{ind}^{*}(\alpha)$ above, we see that the a.e. value of ice on $X_{\alpha}$ is greater than 4 unless the partial quotients $a_{k}$ are eventually 1 . Lebesgue almost every slope $\alpha \in(0,1)$ has unbounded partial quotients, and thus, for Lebesgue a.e. $\alpha$, ice is a.e. infinite on $X_{\alpha}$.

2.3. An additive $S$-adic representation. Let $\omega \in\{0,1\}^{\mathbb{N}}$ be a Sturmian sequence of slope $\alpha$. Exactly one of the words $i i(i \in\{0,1\})$ is a factor of $\omega$ and there is a unique sequence $\omega^{\prime}$ such that $\omega=T^{b}\left(\tau_{i}\left(\omega^{\prime}\right)\right)$, where $b=0$ if $\omega$ begins in $i$ and $b=1$ otherwise. The map $\omega \mapsto \omega^{\prime}$ on $X_{\alpha}$ is really just induction on the longer of the two intervals in the associated two-interval exchange. Specifically, suppose $\omega$ codes the orbit of a point $x$; if $x$ is in the longer interval then $\omega^{\prime}$ codes the orbit of $x$ in the induced interval exchange, and if $x$ is in the other (shorter) interval then $\omega^{\prime}$ codes the orbit of the preimage of $x$ (which is in the longer interval) in the induced interval exchange. With this interpretation it is clear that $\omega^{\prime}$ is also Sturmian. Thus we may iterate this "desubstitution" process to obtain our additive $S$-adic expansion:

Proposition 2.3. Let $\omega$ be a Sturmian sequence. There exist a sequence of Sturmian sequences $\left(\omega^{(n)}\right)_{n \geq 1}$ and two sequences $\left(b_{n}\right)_{n \geq 1},\left(i_{n}\right)_{n \geq 1}$ with values in $\{0,1\}$ such that

(1) $\omega=T^{b_{1}} \circ \tau_{i_{1}} \circ \cdots \circ T^{b_{n}} \circ \tau_{i_{n}}\left(\omega^{(n)}\right)$ for each $n$,

(2) $\left(i_{n}\right)$ is not eventually constant,

(3) if $i_{n}=i_{n+1}$ and $b_{n+1}=0$ then $b_{n}=0$,

(4) if $i_{n} \neq i_{n+1}$ then $b_{n}$ and $b_{n+1}$ are not both 1 .

Proof. The induction process described above gives us the three sequences satisfying assertion (1). If $\left(i_{n}\right)_{n \geq 1}$ were eventually constant, say $i_{n}=i$ for all $n \geq N$, then $\omega$ would contain arbitrary powers of $\tau_{i_{1}} \circ \cdots \circ \tau_{i_{N}}(i)$, which is impossible since $\omega$ is Sturmian.

Assertions (3) and (4) are easily deduced from the facts that $\omega_{0}^{(n)}$ is the first letter of $T^{b_{n+1}} \circ \tau_{i_{n+1}}$, i.e., 


$$
\omega_{0}^{(n)}= \begin{cases}i_{n+1} & \text { if } b_{n+1}=0, \\ \bar{\imath}_{n+1} & \text { if } b_{n+1}=1,\end{cases}
$$

and

$$
b_{n}=1 \Rightarrow \omega_{0}^{(n)}=\bar{\imath}_{n}
$$

It is helpful to think of $T^{b_{1}} \circ \tau_{i_{1}} \circ \cdots \circ T^{b_{n}} \circ \tau_{i_{n}}$ as a composition of "inflations" (the $\tau_{i_{m}}$ ) and "cuts" (the $T^{b_{m}}$ ) where the amount cut after applying $\tau_{i_{m}}$ to $\omega^{(m)}$ is less than the inflated image of the first letter of $\omega^{(m)}$, i.e., $b_{m}<\left|\tau_{i_{m}}\left(\omega_{0}^{(m)}\right)\right|$. Extending this notion of $T$ as the map which cuts off the first letter of a sequence, we shall abuse notation slightly and write $T w$ for the suffix of a word $w$ obtained by deleting the first letter. Let us note that, by definition,

$$
\left|T^{b_{1}} \circ \tau_{i_{1}} \circ \cdots \circ T^{b_{n}} \circ \tau_{i_{n}}\left(\omega_{0}^{(n)}\right)\right| \geq 1 \quad \text { for all } n,
$$

hence

$$
\begin{aligned}
T^{b_{1}} \circ \tau_{i_{1}} \circ & \cdots \circ T^{b_{n}} \circ \tau_{i_{n}}\left(\omega^{(n)}\right) \\
& =T^{b_{1}} \circ \tau_{i_{1}} \circ \cdots \circ T^{b_{n}} \circ \tau_{i_{n}}\left(\omega_{0}^{(n)}\right) \star \tau_{i_{1}} \circ \cdots \circ \tau_{i_{n}}\left(\left(\omega_{k}^{(n)}\right)_{k \geq 1}\right),
\end{aligned}
$$

where, for clarity, we have written $\star$ for concatenation. It is possible that

$$
\left|T^{b_{1}} \circ \tau_{i_{1}} \circ \cdots \circ T^{b_{n}} \circ \tau_{i_{n}}\left(\omega_{0}^{(n)}\right)\right|=1 \quad \text { for all } n \text {. }
$$

This happens, for example, when $i_{n}=b_{n}=n \bmod 2$ for all $n$.

The following useful lemma can be proved by straightforward induction.

LEMMA 2.4. If $v$ and $v^{\prime}$ are sequences in $\{0,1\}$ beginning in different letters and $\tau$ is any composition of the $\tau_{i}$ then the longest common prefix of $\tau(v)$ and $\tau\left(v^{\prime}\right)$ has length $|\tau(01)|-2$.

We next show that what we have is indeed an additive $S$-adic expansion in the sense of $[21,23]$. The important thing is that the sequences $\left(i_{n}\right)_{n \geq 1}$ and $\left(b_{n}\right)_{n \geq 1}$ entirely determine $\omega$; we do not need to keep track of the $\omega^{(\bar{n})}$.

Proposition 2.5. Every pair of sequences $\left(i_{n}\right)_{n \geq 1},\left(b_{n}\right)_{n \geq 1}$ with values in $\{0,1\}$ satisfying (2)-(4) of Proposition 2.3 is the additive $S$-adic expansion of a unique Sturmian sequence.

Proof. Suppose $\left(i_{n}\right),\left(b_{n}\right)$ satisfy (2)-(4) of Proposition 2.3. If $v, v^{\prime} \in$ $\{0,1\}^{\mathbb{N}}$ then it follows from Lemma 2.4 and the previous remarks on cuts and inflations that $T^{b_{1}} \circ \tau_{i_{1}} \circ \cdots \circ T^{b_{n}} \circ \tau_{i_{n}}(v)$ and $T^{b_{1}} \circ \tau_{i_{1}} \circ \cdots \circ T^{b_{n}} \circ \tau_{i_{n}}\left(v^{\prime}\right)$ have a common prefix of length at least $\left|\tau_{i_{1}} \circ \tau_{i_{2}} \circ \cdots \circ \tau_{i_{n}}\left(i_{n}\right)\right|-1$, which tends to infinity as $n$ tends to infinity. Thus $\bigcap_{n=1}^{\infty} T^{b_{1}} \circ \tau_{i_{1}} \circ \cdots \circ T^{b_{n}} \circ \tau_{i_{n}}\left(\{0,1\}^{\mathbb{N}}\right)$ consists of a single point, $\omega$. We claim that $\omega$ is Sturmian. Indeed, if $v$ is any Sturmian sequence then

$$
\omega=\lim _{n \rightarrow \infty} T^{b_{1}} \circ \tau_{i_{1}} \circ \cdots \circ T^{b_{n}} \circ \tau_{i_{n}}(v) .
$$


The morphisms $\tau_{0}$ and $\tau_{1}$ are Sturmian (i.e., they take Sturmian sequences to Sturmian sequences, see [30]) and the complexity of a limit is less than or equal to the limit of the complexities, hence $\omega$ has complexity $p(n) \leq n+1$ and is therefore either Sturmian or eventually periodic. It follows from the fact that $\left(i_{n}\right)_{n \geq 1}$ is not eventually constant that $\omega$ is not eventually periodic, so $p(n) \geq n+1$ and $\omega$ is Sturmian. One checks by induction that $\omega$ has $\left(i_{n}\right)_{n \geq 1},\left(b_{n}\right)_{n \geq 1}$ as its $S$-adic expansion.

Such an expansion will be called the additive Ostrowski $S$-adic expansion associated with the sequence $\omega$. We will see below that Ostrowski expansions in the sense of [35] appear in a natural way when one considers a multiplicative version of these expansions.

2.4. A multiplicative $S$-adic expansion. A more compact version of the additive $S$-adic representation is desirable. As a sequence in $\{0,1\}$ we can write

$$
i_{1} i_{2} \ldots=0^{a_{1}} 1^{a_{2}} 0^{a_{3}} 1^{a_{4}} \ldots
$$

with $a_{i} \geq 1$ for $i \geq 2$. Let $s_{k}=\sum_{j=1}^{k} a_{j}$ and $c_{k}=\sum_{n=s_{k-1}+1}^{s_{k}} b_{n}$. For all $n \geq 1$ we have $0 \leq c_{n} \leq a_{n}$ and if $c_{n+1}=a_{n+1}$ then $c_{n}=0$. We also have

$$
b_{1} b_{2} \ldots=0^{a_{1}-c_{1}} 1^{c_{1}} 0^{a_{2}-c_{2}} 1^{c_{2}} \ldots
$$

and for $k>0$,

$\omega=\tau_{0}^{a_{1}-c_{1}} \circ\left(T \circ \tau_{0}\right)^{c_{1}} \circ \tau_{1}^{a_{2}-c_{2}} \circ\left(T \circ \tau_{1}\right)^{c_{2}} \circ \cdots \circ \tau_{k-1 \bmod 2}^{a_{k}-c_{k}} \circ\left(T \circ \tau_{k-1 \bmod 2}\right)^{c_{k}}\left(\omega^{\left(s_{k}\right)}\right)$.

To avoid cumbersome notation we shall henceforth write $\tau_{n}$ for $\tau_{n} \bmod 2$. We can further simplify to obtain

$$
\omega=T^{c_{1}} \tau_{0}^{a_{1}} \circ T^{c_{2}} \tau_{1}^{a_{2}} \circ T^{c_{3}} \tau_{0}^{a_{3}} \circ \cdots \circ T^{c_{k}} \tau_{k-1}^{a_{k}}\left(\omega^{\left(s_{k}\right)}\right) .
$$

Let $\alpha=\left[0 ; a_{1}+1, a_{2}, a_{3}, \ldots\right]$. Set

$$
\begin{array}{ll}
p_{0}=0, & q_{0}=1, \\
p_{1}=1, & q_{1}=a_{1}+1,
\end{array}
$$

and for $k \geq 2$,

$$
p_{k}=a_{k} p_{k-1}+p_{k-2}, \quad q_{k}=a_{k} q_{k-1}+q_{k-2} .
$$

Set $\delta_{-1}=1-\alpha$, and for $k \geq 0$ put $\delta_{k}=\left|q_{k} \alpha-p_{k}\right|=(-1)^{k}\left(q_{k} \alpha-p_{k}\right)$. One has

$$
\forall k \in \mathbb{N}, \quad \delta_{k-1}=a_{k+1} \delta_{k}+\delta_{k+1} .
$$

The continued fraction convergents of $\alpha$ are the rational numbers $p_{k} / q_{k}$, which, as the name suggests, converge to $\alpha$. The convergents are, in a sense, the best possible rational approximations to $\alpha$. The following lemma can be proved by straightforward induction. 
LEMMA 2.6. Write $|w|_{j}$ for the number of occurrences of the letter $j$ in the word $w$. Then for $i \in\{0,1\}$,

$$
\begin{aligned}
\left(\left|\tau_{0}^{a_{1}} \circ \cdots \circ \tau_{k-1}^{a_{k}}(i)\right|_{0},\left|\tau_{0}^{a_{1}} \circ \cdots \circ \tau_{k-1}^{a_{k}}(i)\right|_{1}\right) & \\
& = \begin{cases}\left(q_{k}-p_{k}, p_{k}\right) & \text { if } i=k \bmod 2, \\
\left(q_{k-1}-p_{k-1}, p_{k-1}\right) & \text { if } i \neq k \bmod 2 .\end{cases}
\end{aligned}
$$

It follows that the slope of $\omega$ is equal to $\lim p_{k} / q_{k}=\alpha$. This means that the $a_{k}$ and hence also the sequence $\left(i_{n}\right)_{n \geq 1}$ are determined by the slope of $\omega$. Translating the condition on the sequences $\left(i_{n}\right)_{n \geq 1}$ and $\left(b_{n}\right)_{n \geq 1}$ to a condition on the $c_{k}$, we have shown how Sturmian sequences of slope $\alpha=$ $\left[0 ; a_{1}+1, a_{2}, \ldots\right]$ are in one-to-one correspondence with sequences $\left(c_{k}\right)_{k \geq 1}$ such that $0 \leq c_{k} \leq a_{k}$ and if $c_{k+1}=a_{k+1}$ then $c_{k}=0$.

Proposition 2.7. Let $\alpha=\left[0 ; a_{1}+1, a_{2}, a_{3}, \ldots\right]$. Let $\omega$ be a Sturmian sequence which codes the orbit of the point $x$ under the action of $R_{\alpha}$ or $\widetilde{R}_{\alpha}$. There exists a sequence of integers $\left(c_{n}\right)_{n \in \mathbb{N}}$ where

$$
\forall n, \quad\left\{\begin{array}{l}
0 \leq c_{n} \leq a_{n}, \\
c_{n+1}=a_{n+1} \Rightarrow c_{n}=0,
\end{array}\right.
$$

and a sequence of Sturmian sequences $\left(v^{(k)}\right)$ such that

$$
\forall k, \quad \omega=T^{c_{1}} \tau_{0}^{a_{1}} \circ T^{c_{2}} \tau_{1}^{a_{2}} \circ T^{c_{3}} \tau_{0}^{a_{3}} \circ \cdots \circ T^{c_{k}} \tau_{k-1}^{a_{k}}\left(v^{(k)}\right),
$$

and

$$
x=\sum_{k=1}^{\infty} c_{k}(-1)^{k-1} \delta_{k-1}=\sum_{k=1}^{\infty} c_{k}\left(q_{k-1} \alpha-p_{k-1}\right) .
$$

Proof. Let us suppose that $\omega$ codes the orbit of $x$ in $[-\alpha, 1-\alpha)$ under the rotation $R_{\alpha}$ with respect to the partition $([-\alpha, 1-2 \alpha),[1-2 \alpha, 1-\alpha)$ ) (the $\widetilde{R}_{\alpha}$ case is similar). We define two-interval exchanges $E^{(n)}$ for $n \geq 0$ as follows:

If $n$ is even then $E^{(n)}:\left[-\delta_{n}, \delta_{n-1}\right) \rightarrow\left[-\delta_{n}, \delta_{n-1}\right)$ is given by

$$
E^{(n)}(z)= \begin{cases}z+\delta_{n} & \text { if } z \in\left[-\delta_{n},-\delta_{n}+\delta_{n-1}\right), \\ z-\delta_{n-1} & \text { if } z \in\left[-\delta_{n}+\delta_{n-1}, \delta_{n-1}\right)\end{cases}
$$

If $n$ is odd then $E^{(n)}:\left[-\delta_{n-1}, \delta_{n}\right) \rightarrow\left[-\delta_{n-1}, \delta_{n}\right)$ is given by

$$
E^{(n)}(z)= \begin{cases}z+\delta_{n-1} & \text { if } z \in\left[-\delta_{n-1},-\delta_{n-1}+\delta_{n}\right), \\ z-\delta_{n} & \text { if } z \in\left[-\delta_{n-1}+\delta_{n}, \delta_{n}\right) .\end{cases}
$$

Note that $E^{(0)}$ equals $R_{\alpha}$. We also define inductively a sequence of points $\left(x^{(n)}\right)_{n \geq 0}$ where

$$
x^{(n)} \in \begin{cases}{\left[-\delta_{n}, \delta_{n-1}\right)} & \text { if } n \text { is even, } \\ {\left[-\delta_{n-1}, \delta_{n}\right)} & \text { if } n \text { is odd }\end{cases}
$$


and a sequence of nonnegative integers $\left(c_{n}\right)_{n \geq 1}$ by setting $x^{(0)}=x$, and for $n>0$ :

If $n$ is even then

$$
c_{n+1}= \begin{cases}0 & \text { if } x^{(n)} \in\left[-\delta_{n}, \delta_{n+1}\right), \\ \left\lfloor\frac{x^{(n)}-\delta_{n+1}}{\delta_{n}}\right\rfloor+1 & \text { if } x^{(n)} \in\left[\delta_{n+1}, \delta_{n-1}\right)\end{cases}
$$

and

$$
x^{(n+1)}=x^{(n)}-c_{n+1} \delta_{n} .
$$

If $n$ is odd then

$$
c_{n+1}= \begin{cases}0 & \text { if } x^{(n)} \in\left[-\delta_{n+1}, \delta_{n}\right), \\ \left\lceil-\frac{x^{(n)}+\delta_{n+1}}{\delta_{n}}\right\rceil & \text { if } x^{(n)} \in\left[-\delta_{n-1},-\delta_{n+1}\right)\end{cases}
$$

and

$$
x^{(n+1)}=x^{(n)}+c_{n+1} \delta_{n} .
$$

Let us check that the admissibility condition (1) holds. We easily see that $c_{k} \leq a_{k}$ for all $k \geq 1$. If $c_{2 k+1} \neq 0$ then $x^{(2 k+1)} \in\left[\delta_{2 k+1}-\delta_{2 k}, \delta_{2 k+1}\right)$, and thus $c_{2 k+2} \neq a_{2 k+2}$. If $c_{2 k+2} \neq 0$ then $x^{(2 k+2)} \in\left[-\delta_{2 k+2},-\delta_{2 k+2}+\delta_{2 k+1}\right)$, and thus $c_{2 k+3} \neq a_{2 k+3}$. thus

Furthermore, for all $n \in \mathbb{N}$ we have $x=x^{(n)}+\sum_{k=0}^{n-1} c_{k+1}(-1)^{k} \delta_{k}$ and

$$
x=\sum_{k=0}^{\infty} c_{k+1}(-1)^{k} \delta_{k} .
$$

We claim that if $n$ is even then $E^{(n+1)}$ is the induced transformation of $E^{(n)}$ on the interval $\left[-\delta_{n}, \delta_{n+1}\right)$. Let us check this. If $z \in\left[-\delta_{n},-\delta_{n}+\delta_{n+1}\right)$ then

$$
E^{(n)}(z)=z+\delta_{n} \in\left[0, \delta_{n+1}\right)
$$

and thus the induced transformation agrees with $E^{(n+1)}$ on $\left[-\delta_{n},-\delta_{n}+\right.$ $\left.\delta_{n+1}\right)$. If $z \in\left[-\delta_{n}+\delta_{n+1}, \delta_{n+1}\right)$ then

$$
\left(E^{(n)}\right)^{k}(z)=z+k \delta_{n} \geq \delta_{n+1} \quad \text { for } 1 \leq k \leq a_{n+1}
$$

and

$$
\left(E^{(n)}\right)^{a_{n+1}+1}=z+\left(a_{n+1}\right) \delta_{n}-\delta_{n-1}=z-\delta_{n+1} \in\left[-\delta_{n}, 0\right),
$$

as desired. One similarly checks that for $n$ odd, $E^{(n+1)}$ is the induced transformation on the interval $\left[-\delta_{n+1}, \delta_{n}\right)$ of the map $E^{(n)}$.

We let $v^{(n)}$ be the Sturmian sequence coding the orbit of $x^{(n)}$ in the two-interval exchange $E^{(n)}$ with respect to the partition $\left(\left[-\delta_{n},-\delta_{n}+\delta_{n-1}\right)\right.$, $\left.\left[-\delta_{n}+\delta_{n-1}, \delta_{n-1}\right)\right)$ if $n$ is even, and to the partition $\left(\left[-\delta_{n-1},-\delta_{n-1}+\delta_{n}\right)\right.$, $\left.\left[-\delta_{n-1}+\delta_{n}, \delta_{n}\right)\right)$ if $n$ is odd. It follows that $v^{(n)}=T^{c_{n+1}} \tau_{n+1}^{a_{n+1}}\left(v^{(n+1)}\right)$ holds for every $n$. 
Remarks. Such an expansion will be called the (multiplicative) Ostrowski $S$-adic expansion associated with the sequence $\omega$. More generally, an expansion of the form

$$
x=\sum_{k=0}^{\infty} c_{k+1}\left(q_{k} \alpha-p_{k}\right)
$$

where the sequence of integer digits $\left(c_{k}\right)_{k \geq 1}$ satisfies the admissibility condition (1), is called an Ostrowski expansion following [35] (see also [10, 18, $19,28,29,26,39,40])$. Note that the characteristic sequence of slope $\alpha$ corresponds to intercept $x=0$, having all $c_{k}$ equal to 0 .

2.5. The Ostrowski odometer. Let $\alpha=\left[0 ; a_{1}+1, a_{2}, \ldots\right]$ and set

$$
K_{\alpha}=\left\{\left(c_{k}\right)_{k \geq 1}: \forall k \geq 1,\left(c_{k} \in \mathbb{N}, 0 \leq c_{k} \leq a_{k}\right) \text { and }\left(c_{k+1}=a_{k+1} \Rightarrow c_{k}=0\right)\right\} .
$$

It is easy to see that

$$
K_{\alpha}=\left\{\left(c_{k}\right)_{k \geq 1}: \forall k \geq 1, c_{k} \in \mathbb{N}, c_{1} q_{0}+\cdots+c_{k} q_{k-1} \leq q_{k}-1\right\} .
$$

Let $c=\left(c_{k}\right)_{k \geq 1} \in K_{\alpha}$, set

$$
D(c)=\left\{k \geq 1: c_{1} q_{0}+\cdots+c_{k} q_{k-1}=q_{k+1}-1\right\},
$$

and put $m(c)=\sup D(c)$ if $D(c)$ is nonempty, and $m(c)=-1$ otherwise. Note that $m(c)=\infty$ if and only if $c$ is of the form

$$
a_{1} 0 a_{3} 0 \ldots \text { or } 0 a_{2} 0 a_{4} \ldots,
$$

and if $m(c)>0$ then

$$
c= \begin{cases}a_{1} 0 a_{3} 0 \ldots a_{m(c)-1} 0 c_{m(c)+1} c_{m(c)+2} \ldots & \text { if } m(c) \text { is even, } \\ 0 a_{2} 0 a_{4} \ldots 0 a_{m(c)-1} 0 c_{m(c)+1} c_{m(c)+2} \ldots & \text { if } m(c) \text { is odd. }\end{cases}
$$

Following [24], one can define on the compact set $K_{\alpha}$ (endowed with the product of the discrete topologies on the finite sets $\left.\left\{0 \leq d \leq a_{k}\right\}\right)$ the addition $\sigma$ by 1 :

$$
\sigma(c)= \begin{cases}0^{m(c)+1}\left(c_{m(c)+1}+1\right) c_{m(c)+2} \cdots & \text { if } m(c)<\infty \\ 0^{\infty} & \text { otherwise }\end{cases}
$$

The map $\sigma$ is called the Ostrowski $\alpha$-odometer. The map $\sigma: K_{\alpha} \rightarrow K_{\alpha}$ is onto and continuous, and $\left(K_{\alpha}, \sigma\right)$ is minimal (for more details, see [24, 7]).

Proposition 2.8. The dynamical systems $\left(K_{\alpha}, \sigma\right)$ and $\left(X_{\alpha}, T\right)$ are topologically conjugate.

Proof. The sets $X_{\alpha}$ and $K_{\alpha}$ are in one-to-one correspondence via the map $\Psi: X_{\alpha} \rightarrow K_{\alpha}, \omega \mapsto\left(c_{k}\right)_{k \geq 1}$, where $\left(c_{k}\right)_{k \geq 1}$ is the Ostrowski $S$-adic expansion of Proposition 2.7. 
Suppose $\omega \in X_{\alpha}$ and $\Psi(\omega)=c$ does not have a tail in common with $a_{1} 0 a_{3} 0 \ldots$ or $0 a_{2} 0 a_{4} \ldots$ Put $m=\max D(c)$ and let $v^{(k)}$ be as in Proposition 2.7. Then $c_{m+1}<a_{m+1}$ and

$$
\begin{aligned}
T(\omega) & =T\left(T^{c_{1}} \tau_{0}^{a_{1}} \circ \cdots \circ T^{c_{m}} \tau_{m-1}^{a_{m}}\left(v^{(m)}\right)\right) \\
& =\tau_{0}^{a_{1}} \circ \cdots \circ \tau_{m-1}^{a_{m}}\left(T v^{(m)}\right) \\
& =\tau_{0}^{a_{1}} \circ \cdots \circ \tau_{m-1}^{a_{m}} \circ T^{c_{m+1}+1} \tau_{m}^{a_{m+1}}\left(v^{(m+1)}\right),
\end{aligned}
$$

whence $\Psi(T \omega)=\sigma(\Psi(\omega))$. This holds for a dense set of $\omega \in X_{\alpha}$.

\subsection{A characterization of primitive substitutive Sturmian se-}

quences. Let $\mathcal{A}$ be a finite alphabet and denote by $\mathcal{A}^{*}$ the free monoid generated by $\mathcal{A}$ with concatenation as the multiplication, i.e., $\mathcal{A}^{*}$ is the set of finite words over the alphabet $\mathcal{A}$. A substitution is a morphism of the free monoid $\mathcal{A}^{*}$ taking each element of $\mathcal{A}$ to a nonempty word. A substitution $\tau$ is primitive if there exists an integer $k$ such that for all letters $a, b$ in the alphabet $\mathcal{A}, a$ is a factor of $\tau^{k}(b)$. A sequence $u$ is primitive substitutive if there exist a primitive substitution $\tau$ over an alphabet $\mathcal{B}$ and a letter-toletter morphism $\varphi: \mathcal{B} \rightarrow \mathcal{A}$ such that $u=\varphi(v)$, where $v=\tau(v) \in \mathcal{B}^{\mathbb{N}}$ is fixed by $\tau$. We shall characterize primitive substitutive Sturmian sequences in this section. For characterizations of Sturmian sequences that are fixed points of substitutions, see $[15,36,44]$. Let us recall a fact about Ostrowski's numeration (see for instance [26]):

Theorem 2.9. Let

$$
x=\sum_{k=1}^{\infty} c_{k+1}\left(q_{k} \alpha-p_{k}\right),
$$

where the sequence $\left(c_{k}\right)_{k \geq 1}$ satisfies the admissibility conditions (1). Suppose $\alpha$ is quadratic. Then $\left(c_{k}\right)_{k \geq 1}$ is eventually periodic if and only if $x \in \mathbb{Q}(\alpha)$.

Let $\omega$ be a uniformly recurrent sequence, and let $h$ be a factor of $\omega$. Recall that a return word to $h$ is a factor $\omega[i, j)$, where $h$ occurs in $\omega$ starting at the $i$ th and $j$ th places and nowhere between. Let $\mathcal{A}_{h}$ be the set of return words to $h$ in $\omega$. A sequence $v$ with the same set of factors as $\omega$ and having $h$ as a prefix can be recoded over the alphabet $\mathcal{A}_{h}$. Indeed we can naturally write the sequence $v$ as a concatenation of return words to $h$ and this decomposition is unique. We enumerate the elements of the set $\mathcal{A}_{h}$ of return words to $h$ in the order of their first appearance in the sequence $\omega$, turning this set into a new alphabet. We then can recode the sequence $v$ over this new alphabet. The recoded sequence, called a derived sequence of $v$, is denoted by $\mathcal{D}_{h}(v)$. One can also associate a derived sequence with a sequence $v$ not having $h$ as a prefix as follows. Let $p$ be a prefix of a return word in $\mathcal{A}_{h}$ such that the sequence $p v$ starts with $h$ and has the same set of factors as $\omega$. We will 
also call a derived sequence the sequence over $\mathcal{A}_{h}$ obtained by coding the sequence $p v$. We will use the following result $[20,25,22]$ :

THEOREM 2.10. A uniformly recurrent sequence is primitive substitutive if and only if the set of derived sequences (up to the alphabet) over all its factors is finite.

Note that an expansion of the form

$\omega=\tau_{0}^{a_{1}-c_{1}} \circ\left(T \circ \tau_{0}\right)^{c_{1}} \circ \tau_{1}^{a_{2}-c_{2}} \circ\left(T \circ \tau_{1}\right)^{c_{2}} \circ \cdots \circ \tau_{k-1}^{a_{k}-c_{k}} \circ\left(T \circ \tau_{k-1}\right)^{c_{k}}\left(\omega^{\left(s_{k}\right)}\right)$ can explicitly be written as a standard $S$-adic expansion, that is, as a limit of the composition of a finite number of substitutions following [23, 21], by introducing the morphisms $\tau_{i}^{\prime}$ for $i \in\{0,1\}$ defined by $\tau_{i}^{\prime}(i)=i$ and $\tau_{i}^{\prime}(j)=j i$ for $j \neq i$. Indeed we have

$$
\omega=\tau_{0}^{a_{1}-c_{1}} \circ\left(\tau_{0}^{\prime}\right)^{c_{1}} \circ \tau_{1}^{a_{2}-c_{2}} \circ\left(\tau_{1}^{\prime}\right)^{c_{2}} \circ \cdots \circ \tau_{k-1}^{a_{k}-c_{k}} \circ\left(\tau_{k-1}^{\prime}\right)^{c_{k}}\left(\omega^{\left(s_{k}\right)}\right) .
$$

Proposition 2.11. A Sturmian sequence $\omega$ of slope $\alpha$ which codes the orbit of $x$ is primitive substitutive if and only if $\alpha$ is a quadratic irrational and $x \in \mathbb{Q}(\alpha)$.

Proof. If $\alpha$ is quadratic and $x \in \mathbb{Q}(\alpha)$, then $\left(a_{k}\right)_{k \geq 1}$ and $\left(c_{k}\right)_{k \geq 1}$ are eventually periodic. The standard $S$-adic expansion above (using the $\tau_{i}$ and $\left.\tau_{i}^{\prime}\right)$ is eventually periodic, and $\omega$ is seen to be primitive substitutive.

Conversely, suppose $\omega$ is primitive substitutive. We will use the notation of Proposition 2.7. The sequences $v^{(k)}$ are derived sequences. More precisely, if $k \bmod 2$ denotes the letter in $\{0,1\}$ with value $k \bmod 2$, then

$$
v^{(k+1)}=\mathcal{D}_{(k \bmod 2)^{a} k_{k+1}}\left((k \bmod 2)^{c_{k+1}} v^{(k)}\right),
$$

where we denote by $(k \bmod 2)^{c_{k+1}} v^{(k)}$ the sequence made of the word $(k \bmod 2)^{c_{k+1}}$ concatenated with the sequence $v^{(k)}$. Indeed, $(k \bmod 2)^{c_{k+1}} 1$ and $(k \bmod 2)^{c_{k+1}+1} 1$ are exactly the two return words of $(k \bmod 2)^{c_{k+1}}$ in $\omega$, the second one corresponding to the interval of induction. The derived sequence of a derived sequence is again a derived sequence (up to the alphabet). Hence following Theorem 2.10, there are two sequences $v^{(k)}$ and $v^{(l)}$ which are equal, hence $\left(a_{k}\right)_{k \geq 1}$ and $\left(c_{k}\right)_{k \geq 1}$ are eventually periodic.

\section{CALCULATING INITIAL POWERS}

The paradigm for our study is that large initial powers of $\omega$ come from large initial powers of the $\omega^{(n)}$. Before giving a more precise statement let us prove a simpler fact. Let $\omega$ be a Sturmian sequence and let $i_{n}, b_{n}, \omega^{(n)}$ be defined as in the previous section. Recall that a word is primitive if it is not an integer power of a shorter word. 
LEMMA 3.1. If $\omega$ begins in a word $w^{r}$ where $r>1,|w|>2$, and $w$ is primitive then there is a prefix $w^{(1)}$ of $\omega^{(1)}$ such that $w$ is a cyclic permutation of $\tau_{i_{1}}\left(w^{(1)}\right)$. Furthermore, $\left|w^{(1)}\right| \geq 2$ and $w^{(1)}$ is primitive.

Proof. If $b_{1}=0$ then $\omega_{0}=\omega_{|w|}=i_{1}$. The only place that $i_{1}$ occurs in the image of a letter under $\tau_{i_{1}}$ is as the first letter. Thus the longest word of the form $\tau_{i_{1}}\left(\omega_{0}^{(1)}\right) \tau_{i_{1}}\left(\omega_{1}^{(1)}\right) \ldots \tau_{i_{1}}\left(\omega_{j}^{(1)}\right)$ which is a prefix of $w$ must in fact be $w$, so that $w^{(1)}=\omega^{(1)}[0, j]$ does the job.

In the case $b_{1}=1$, we have $\tau_{i_{1}}\left(\omega^{(1)}\right)=i_{1} \omega$, and $\omega_{0}=\omega_{|w|}=\bar{\imath}_{1}$. Since no sequence in the image of $\tau_{i_{1}}$ can have $\bar{\imath}_{1} \bar{\imath}_{1}$ as a factor, it must be that $\omega_{|w|-1}=i_{1}$. The same argument used in the first case produces a prefix $w^{(1)}$ of $\omega^{(1)}$ for which $\tau_{i_{1}}\left(w^{(1)}\right)=i_{1} w[0,|w|-2]$, and $i_{1} w[0,|w|-2]$ is a cyclic permutation of $w$.

Now $\left|\tau_{i_{1}}(u)\right| \leq 2|u|$ for any word $u$, and $\left|\tau_{i_{1}}\left(w^{(1)}\right)\right|=|w|>2$, so we must have $\left|w^{(1)}\right| \geq 2$, and if $w^{(1)}$ were an integer power of some shorter word then $w$ would be also, contrary to the hypothesis.

We are now prepared to prove an important fact about initial powers. Let us recall that for all $k>0, s_{k}=\sum_{j=1}^{k} a_{j}$, and

$$
\begin{aligned}
\omega & =\tau_{0}^{a_{1}-c_{1}} \circ\left(T \circ \tau_{0}\right)^{c_{1}} \circ \tau_{1}^{a_{2}-c_{2}} \circ\left(T \circ \tau_{1}\right)^{c_{2}} \circ \cdots \circ \tau_{k-1}^{a_{k}-c_{k}} \circ\left(T \circ \tau_{k-1}\right)^{c_{k}}\left(\omega^{\left(s_{k}\right)}\right) \\
& =T^{c_{1}} \tau_{0}^{a_{1}} \circ T^{c_{2}} \tau_{1}^{a_{2}} \circ T^{c_{3}} \tau_{0}^{a_{3}} \circ \cdots \circ T^{c_{k}} \tau_{k-1}^{a_{k}}\left(\omega^{\left(s_{k}\right)}\right) .
\end{aligned}
$$

Proposition 3.2. Suppose $\omega$ begins in a word $w$ to power $r \geq 2$, where $|w| \geq 2$, and $w$ is primitive. Then there is a nonnegative integer $m$ such that $w$ is a cyclic permutation of $\tau_{i_{1}} \circ \cdots \circ \tau_{i_{m}}(01)$, and $\omega^{(m)}$ begins in 01 or 10 to power $>\lfloor r\rfloor-1$. Furthermore, $m$ is one of the numbers $s_{k}-1$ or $s_{k}-c_{k}-1$. If $r \geq 3$ then $m$ is one of the numbers $s_{k}-1$.

Proof. Let $w^{(1)}$ be the prefix of $\omega^{(1)}$ given by Lemma 3.1. If $\left|w^{(1)}\right|>2$ and the prefix power of $w^{(1)}$ in $\omega^{(1)}$ is $>1$ then we can apply the lemma again to get a prefix $w^{(2)}$ of $\omega^{(2)}$. Continue in this way as long as possible, at the $n$th step obtaining a prefix $w^{(n)}$ of $\omega^{(n)}$ for which $\tau_{i_{n}}\left(w^{(n)}\right)$ is a cyclic permutation of $w^{(n-1)}$, stopping after $m$ steps when either $\left|w^{(m)}\right|=2$ or the prefix power $r^{\prime}$ of $w^{(m)}$ in $\omega^{(m)}$ is 1 . We shall show that $r^{\prime}>1$ and $\left|w^{(m)}\right|=2$, from which it follows that $w^{(m)}$ is 01 or 10 since $w^{(m)}$ is primitive, and hence $w$ is a cyclic permutation of $\tau_{i_{1}} \circ \cdots \circ \tau_{i_{m}}(01)$.

Write $\left(w^{(m)}\right)^{\infty}$ for the infinite periodic word $w^{(m)} w^{(m)} w^{(m)} \ldots$ The longest common prefix shared by $\left(w^{(m)}\right)^{\infty}$ and $\omega^{(m)}$ is $\left(w^{(m)}\right)^{r^{\prime}}$, so by Lemma 2.4 the longest common prefix of

$$
\tau_{i_{1}} \circ \cdots \circ \tau_{i_{m}}\left(\left(w^{(m)}\right)^{\infty}\right) \text { and } \tau_{i_{1}} \circ \cdots \circ \tau_{i_{m}}\left(\omega^{(m)}\right)
$$

has length

$$
\left|\tau_{i_{1}} \circ \cdots \circ \tau_{i_{m}}\left(\left(w^{(m)}\right)^{r^{\prime}}\right)\right|+\left|\tau_{i_{1}} \circ \cdots \circ \tau_{i_{m}}(01)\right|-2<\left|\tau_{i_{1}} \circ \cdots \circ \tau_{i_{m}}\left(\left(w^{(m)}\right)^{r^{\prime}+1}\right)\right|,
$$


since $w^{(m)}$ must contain both a 0 and a 1 , by primitivity of $w^{(m)}$, and since $\left|w^{(m)}\right|>1$ in view of Lemma 3.1.

On the other hand,

$$
T^{b_{1}} \circ \tau_{i_{1}} \circ \cdots \circ T^{b_{m}} \circ \tau_{i_{m}}\left(\left(w^{(m)}\right)^{\infty}\right) \quad \text { and } \quad T^{b_{1}} \circ \tau_{i_{1}} \circ \cdots \circ T^{b_{m}} \circ \tau_{i_{m}}\left(\omega^{(m)}\right)
$$

have $w^{r}$ as their longest common prefix and thus

$$
\tau_{i_{1}} \circ \cdots \circ \tau_{i_{m}}\left(\left(w^{(m)}\right)^{\infty}\right) \text { and } \tau_{i_{1}} \circ \cdots \circ \tau_{i_{m}}\left(\omega^{(m)}\right)
$$

have a common prefix of length $\geq r|w|$. Putting these inequalities together we get

$$
\left|\tau_{i_{1}} \circ \cdots \circ \tau_{i_{m}}\left(\left(w^{(m)}\right)^{r^{\prime}+1}\right)\right|>r\left|\tau_{i_{1}} \circ \cdots \circ \tau_{i_{m}}\left(w^{(m)}\right)\right|,
$$

from which we may deduce that $\left\lfloor r^{\prime}\right\rfloor \geq\lfloor r\rfloor-1$ and if $r$ or $r^{\prime}$ is an integer then $r^{\prime}>r-1$. Thus $r^{\prime}>1$ and hence $\left|w^{(m)}\right|=2$ as claimed. This shows that $\omega^{(m)}$ begins in 01 or 10 to power $r^{\prime}>\lfloor r\rfloor-1$.

Let us now examine $m$ more closely. We know that $\omega^{(m)}$ begins in 010 or 101 ; indeed $w^{(m)}=01$ or 10 and $r^{\prime}>1$. By symmetry we need only consider the former possibility.

CASE 1: $i_{m+1}=0$. Since $\omega^{(m)}$ begins in 01 , we have $b_{m+1}=0$ and $\omega^{(m+1)}$ must begin in 1 . If $i_{m+2}=0$ then this means $b_{m+2}=1$, i.e., $m$ is one of the numbers $s_{k}-c_{k}-1$, where $0<c_{k}<a_{k}$. Otherwise $i_{m+2}=1$ and $m$ is one of the $s_{k}-1$.

CASE $2: i_{m+1}=1$. Then $b_{m+1}=1$ and $\omega^{(m+1)}$ begins in 00 , which means $i_{m+2}=0$, and hence $m$ is one of the $s_{k}-1$.

From the first case we see that if $m$ is one of the numbers $s_{k}-c_{k}-1$ $\left(0<c_{k}<a_{k}\right)$ then $\omega^{(m)}=\tau_{0} \circ T \circ \tau_{0}\left(\omega^{(m+2)}\right)$ and $\omega^{(m+2)}$ begins in 1 , and thus in 10 since 11 does not occur as a factor in $\omega^{(m+2)}$ (one has $i_{m+2}=0$ ), which is enough to guarantee that $\omega^{(m)}$ begins in 0100 , i.e., $r^{\prime}=3 / 2$. This cannot happen if $r \geq 3$, since $\left\lfloor r^{\prime}\right\rfloor \geq\lfloor r\rfloor-1$.

Now that we know where prefix powers $r \geq 2$ in $\omega$ come from we can compute them exactly.

Proposition 3.3. Let $w$ and $r$ be as in Proposition 3.2 and let $m, \omega^{(m)}$, and $w^{(m)}$ be as in its proof. Assume that $r$ is the largest power of $w$ which is a prefix of $\omega$. Then

$$
r=\left\{\begin{array}{lr}
\mathbf{1}_{a_{k+2}=c_{k+2}}+\frac{\sum_{j=1}^{k+1}\left(a_{j}-c_{j}\right) q_{j-1}}{q_{k}} & \text { if } m=s_{k}-1, \\
1+\frac{\sum_{j=1}^{k}\left(a_{j}-c_{j}\right) q_{j-1}}{q_{k}-c_{k} q_{k-1}} & \text { if } m=s_{k}-c_{k}-1 \\
\text { with } 0<c_{k}<a_{k},
\end{array}\right.
$$

where $\mathbf{1}_{a_{k+2}=c_{k+2}}$ is 1 if $a_{k+2}=c_{k+2}$ and 0 otherwise. 
Conversely, for each $k, \omega$ begins in a cyclic permutation of $\tau_{0}^{a_{1}} \circ \tau_{1}^{a_{2}} \circ$ .. $\tau_{k-1}^{a_{k}}(01)$ with prefix power

$$
\mathbf{1}_{a_{k+2}=c_{k+2}}+\frac{\sum_{j=1}^{k+1}\left(a_{j}-c_{j}\right) q_{j-1}}{q_{k}}
$$

and for each $k$ such that $0<c_{k}<a_{k}, \omega$ begins in a cyclic permutation of the word $\tau_{0}^{a_{1}} \circ \tau_{1}^{a_{2}} \circ \cdots \circ \tau_{k-1}^{a_{k}-c_{k}-1}(01)$ with prefix power

$$
1+\frac{\sum_{j=1}^{k}\left(a_{j}-c_{j}\right) q_{j-1}}{q_{k}-c_{k} q_{k-1}} .
$$

Before proving the proposition let us state a lemma, closely related to Lemma 2.6, to be used in the calculation. It is proved easily by induction.

LEMma 3.4. Let $k>0$ and set $i=k \bmod 2$. Then

$$
\begin{aligned}
& \left|\tau_{0}^{a_{1}} \circ \tau_{1}^{a_{2}} \circ \cdots \circ \tau_{k-1}^{a_{k}}(i \bar{\imath})\right|=q_{k}+q_{k-1}=2+\sum_{j=1}^{k} a_{j} q_{j-1}, \\
& \left|\tau_{0}^{a_{1}} \circ \tau_{1}^{a_{2}} \circ \cdots \circ \tau_{k-1}^{a_{k}}(i)\right|=q_{k}, \\
& \left|T^{c_{1}} \circ \tau_{0}^{a_{1}} \circ \cdots \circ T^{c_{k}} \circ \tau_{k-1}^{a_{k}}(i)\right|=q_{k}-\sum_{j=1}^{k} c_{j} q_{j-1}, \\
& \left|T^{c_{1}} \circ \tau_{0}^{a_{1}} \circ \cdots \circ T^{c_{k}} \circ \tau_{k-1}^{a_{k}}(i \bar{\imath})\right|=2+\sum_{j=1}^{k}\left(a_{j}-c_{j}\right) q_{j-1} .
\end{aligned}
$$

Proof of Proposition 3.3. First suppose $m=s_{k}-1$. Set $i=k \bmod 2$. The sequence $\omega^{\left(s_{k}\right)}$ begins in $i^{\mathbf{1}_{a_{k+2}=c_{k+2}}+a_{k+1}-c_{k+1}} \bar{\imath}$. Indeed, $\omega^{\left(s_{k}\right)}=T^{c_{k+1}} \circ$ $\tau_{i}^{a_{k+1}} \omega^{\left(s_{k+1}\right)}$; if $a_{k+2} \neq c_{k+2}$, then $\omega^{\left(s_{k+1}\right)}$ begins in $\bar{\imath}$ and $\omega^{\left(s_{k}\right)}$ begins in $i^{a_{k+1}-c_{k+1}} \bar{\imath}$; if $a_{k+2}=c_{k+2}$, then $\omega^{\left(s_{k+1}\right)}$ begins in $i \bar{\imath}$ and since $c_{k+1}=0$, $\omega^{\left(s_{k}\right)}$ begins in $i^{1+a_{k+1}} \bar{\imath}$. The longest common prefix of

$\omega=T^{c_{1}} \circ \tau_{0}^{a_{1}} \circ \cdots \circ T^{c_{k}} \circ \tau_{k-1}^{a_{k}}\left(\omega^{\left(s_{k}\right)}\right)$ and $T^{c_{1}} \circ \tau_{0}^{a_{1}} \circ \cdots \circ T^{c_{k}} \circ \tau_{k-1}^{a_{k}}\left(i^{\infty}\right)$

has the following length by Lemma 2.4:

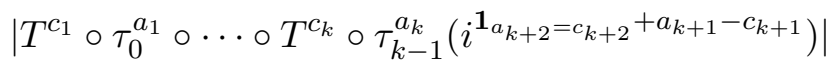

$$
\begin{aligned}
& +\left|\tau_{0}^{a_{1}} \circ \tau_{1}^{a_{2}} \circ \cdots \circ \tau_{k-1}^{a_{k}}(i \bar{\imath})\right|-2 \\
& =\left(\mathbf{1}_{a_{k+2}=c_{k+2}}+a_{k+1}-c_{k+1}-1\right)\left|\tau_{0}^{a_{1}} \circ \tau_{1}^{a_{2}} \circ \cdots \circ \tau_{k-1}^{a_{k}}(i)\right| \\
& +\left|T^{c_{1}} \circ \tau_{0}^{a_{1}} \circ \cdots \circ T^{c_{k}} \circ \tau_{k-1}^{a_{k}}(i)\right|+\left|\tau_{0}^{a_{1}} \circ \cdots \circ \tau_{k-1}^{a_{k}}(i \bar{\imath})\right|-2 \\
& =\left(\mathbf{1}_{a_{k+2}=c_{k+2}}+a_{k+1}-c_{k+1}\right)\left|\tau_{0}^{a_{1}} \circ \tau_{1}^{a_{2}} \circ \cdots \circ \tau_{k-1}^{a_{k}}(i)\right| \\
& +\left|T^{c_{1}} \circ \tau_{0}^{a_{1}} \circ \cdots \circ T^{c_{k}} \circ \tau_{k-1}^{a_{k}}(i \bar{\imath})\right|-2
\end{aligned}
$$




$$
\begin{aligned}
& =\sum_{j=1}^{k}\left(a_{j}-c_{j}\right) q_{j-1}+\left(\mathbf{1}_{a_{k+2}=c_{k+2}}+a_{k+1}-c_{k+1}\right) q_{k} \\
& =\sum_{j=1}^{k+1}\left(a_{j}-c_{j}\right) q_{j-1}+q_{k}\left(\mathbf{1}_{a_{k+2}=c_{k+2}}\right) .
\end{aligned}
$$

Thus $\omega$ begins in a cyclic permutation of $\tau_{0}^{a_{1}} \circ \tau_{1}^{a_{2}} \circ \cdots \circ \tau_{k-1}^{a_{k}}(i)$ to power

$$
\frac{\sum_{j=1}^{k+1}\left(a_{j}-c_{j}\right) q_{j-1}+q_{k} \mathbf{1}_{a_{k+2}=c_{k+2}}}{\left|\tau_{0}^{a_{1}} \circ \tau_{1}^{a_{2}} \circ \cdots \circ \tau_{k-1}^{a_{k}}(i)\right|}=\mathbf{1}_{a_{k+2}=c_{k+2}}+\frac{\sum_{j=1}^{k+1}\left(a_{j}-c_{j}\right) q_{j-1}}{q_{k}} .
$$

Since $\tau_{k-1}(i)=\bar{\imath} i$, this power is exactly the value of $r$.

Next we consider the case $m=s_{k}-c_{k}-1$ with $0<c_{k}<a_{k}$. Again, set $i=k \bmod 2$. From

$\omega=\tau_{0}^{a_{1}-c_{1}} \circ\left(T \circ \tau_{0}\right)^{c_{1}} \circ \tau_{1}^{a_{2}-c_{2}} \circ\left(T \circ \tau_{1}\right)^{c_{2}} \circ \cdots \circ \tau_{k-1}^{a_{k}-c_{k}} \circ\left(T \circ \tau_{k-1}\right)^{c_{k}}\left(\omega^{\left(s_{k}\right)}\right)$,

it is easy to see that $\omega^{\left(s_{k}-c_{k}\right)}$ begins in $i \bar{l}$. Indeed, since $c_{k} \neq 0$, we have $c_{k+1} \neq a_{k+1}$, and $\omega^{\left(s_{k}\right)}$ begins in $i$, so $\omega^{\left(s_{k}-c_{k}\right)}=\left(T \circ \tau_{k-1}\right)^{c_{k}}\left(\omega^{\left(s_{k}\right)}\right)$ begins in $i$, and then in $i \bar{l}$, since $i i$ does not occur in $\omega^{\left(s_{k}-c_{k}\right)}$. We thus deduce that the longest common prefix of

$$
\omega=T^{c_{1}} \circ \tau_{0}^{a_{1}} \circ \cdots \circ T^{c_{k-1}} \circ \tau_{k-2}^{a_{k-1}} \circ \tau_{k-1}^{a_{k}-c_{k}}\left(\omega^{\left(s_{k}-c_{k}\right)}\right)
$$

and

$$
T^{c_{1}} \circ \tau_{0}^{a_{1}} \circ \cdots \circ T^{c_{k-1}} \circ \tau_{k-2}^{a_{k-1}} \circ \tau_{k-1}^{a_{k}-c_{k}}\left(i^{\infty}\right)
$$

has length

$$
\begin{aligned}
& \left|T^{c_{1}} \circ \tau_{0}^{a_{1}} \circ \cdots \circ T^{c_{k-1}} \circ \tau_{k-2}^{a_{k-1}} \circ \tau_{k-1}^{a_{k}-c_{k}}(i)\right| \\
& +\left|\tau_{0}^{a_{1}} \circ \tau_{1}^{a_{2}} \circ \cdots \circ \tau_{k-2}^{a_{k-1}} \circ \tau_{k-1}^{a_{k}-c_{k}}(i \bar{\imath})\right|-2 \\
& =\left|T^{c_{1}} \circ \tau_{0}^{a_{1}} \circ \cdots \circ T^{c_{k-1}} \circ \tau_{k-2}^{a_{k-1}}\left(\bar{\imath}^{a_{k}-c_{k}} i\right)\right|+\left|\tau_{0}^{a_{1}} \circ \tau_{1}^{a_{2}} \circ \cdots \circ \tau_{k-2}^{a_{k-1}}(\bar{\imath})\right| \\
& +\left|\tau_{0}^{a_{1}} \circ \tau_{1}^{a_{2}} \circ \cdots \circ \tau_{k-2}^{a_{k-1}} \circ \tau_{k-1}^{a_{k}-c_{k}}(i)\right|-2 \\
& =\left|T^{c_{1}} \circ \tau_{0}^{a_{1}} \circ \cdots \circ T^{c_{k-1}} \circ \tau_{k-2}^{a_{k-1}}(i \bar{\imath})\right|+\left(a_{k}-c_{k}\right)\left|\tau_{0}^{a_{1}} \circ \tau_{1}^{a_{2}} \circ \cdots \circ \tau_{k-2}^{a_{k-1}}(\bar{\imath})\right| \\
& +\left|\tau_{0}^{a_{1}} \circ \tau_{1}^{a_{2}} \circ \cdots \circ \tau_{k-2}^{a_{k-1}} \circ \tau_{k-1}^{a_{k}-c_{k}}(i)\right|-2 \\
& =\sum_{j=1}^{k-1}\left(a_{j}-c_{j}\right) q_{j-1}+\left(a_{k}-c_{k}\right) q_{k-1}+\left|\tau_{0}^{a_{1}} \circ \tau_{1}^{a_{2}} \circ \cdots \circ \tau_{k-2}^{a_{k-1}} \circ \tau_{k-1}^{a_{k}-c_{k}}(i)\right| \\
& =\sum_{j=1}^{k}\left(a_{j}-c_{j}\right) q_{j-1}+\left|\tau_{0}^{a_{1}} \circ \tau_{1}^{a_{2}} \circ \cdots \circ \tau_{k-2}^{a_{k-1}} \circ \tau_{k-1}^{a_{k}-c_{k}}(i)\right|
\end{aligned}
$$


We also have

$$
\left|\tau_{0}^{a_{1}} \circ \tau_{1}^{a_{2}} \circ \cdots \circ \tau_{k-2}^{a_{k-1}} \circ \tau_{k-1}^{a_{k}-c_{k}}(i)\right|=q_{k}-c_{k} q_{k-1}
$$

and thus $\omega$ begins in a cyclic permutation of $\tau_{0}^{a_{1}} \circ \tau_{1}^{a_{2}} \circ \cdots \circ \tau_{k-2}^{a_{k-1}} \circ \tau_{k-1}^{a_{k}-c_{k}}(i)$ to power

$$
1+\frac{\sum_{j=1}^{k}\left(a_{j}-c_{j}\right) q_{j-1}}{q_{k}-c_{k} q_{k-1}}
$$

As in the first case, this is exactly the value of $r$.

To prove the "conversely" part of the proposition, simply note that the formulas for the lengths above do not depend on $r$ or $m$ at all.

Corollary 3.5 .

$\operatorname{ice}(\omega)=\limsup _{k \rightarrow \infty} \max \left(\frac{\sum_{j=1}^{k+1}\left(a_{j}-c_{j}\right) q_{j-1}}{q_{k}}, 1+\frac{\sum_{j=1}^{k}\left(a_{j}-c_{j}\right) q_{j-1}}{q_{k}-c_{k} q_{k-1}}\right)$.

Proof. Set

$x(k)=\mathbf{1}_{a_{k+2}=c_{k+2}}+\frac{\sum_{j=1}^{k+1}\left(a_{j}-c_{j}\right) q_{j-1}}{q_{k}}, \quad y(k)=1+\frac{\sum_{j=1}^{k}\left(a_{j}-c_{j}\right) q_{j-1}}{q_{k}-c_{k} q_{k-1}}$.

One has, from Proposition 3.3,

$$
\operatorname{ice}(\omega)=\max \left(\limsup _{k \rightarrow \infty} x(k), \limsup _{k \rightarrow \infty, 0<c_{k}<a_{k}} y(k)\right) .
$$

Observe that

- If $c_{k}=a_{k}$ then $y(k)=x(k-2)$. Thus, if $c_{k+2}=a_{k+2}$ then $x(k)=$ $y(k+2)$.

- If $c_{k}=0$ and $c_{k+1}=a_{k+1}$ then $y(k)<y(k+1)=x(k-1)$.

- If $c_{k}=0$ and $c_{k+1}<a_{k+1}$ then $y(k) \leq x(k)$.

The conclusion follows from these observations.

\section{ICE FOR SOME SPECIAL STURMIAN SEQUENCES}

4.1. Notation. In all that follows,

$$
\begin{aligned}
x(k) & =\mathbf{1}_{a_{k+2}=c_{k+2}}+\frac{\sum_{j=1}^{k+1}\left(a_{j}-c_{j}\right) q_{j-1}}{q_{k}}, \\
x^{\prime}(k) & =\frac{\sum_{j=1}^{k+1}\left(a_{j}-c_{j}\right) q_{j-1}}{q_{k}}, \\
y(k) & =1+\frac{\sum_{j=1}^{k}\left(a_{j}-c_{j}\right) q_{j-1}}{q_{k}-c_{k} q_{k-1}} .
\end{aligned}
$$

One has

$$
\operatorname{ice}(\omega)=\limsup _{k \rightarrow \infty} \max (x(k), y(k))=\limsup _{k \rightarrow \infty} \max \left(x^{\prime}(k), y(k)\right) .
$$


4.2. Characteristic sequence. Recall that the characteristic sequence $\omega$ of slope $\alpha$ is the sequence obtained by setting all of the $c_{j}$ equal to 0 . We now prove Theorem 1.2 that we recall below.

TheOREM 1.2. Let $\omega$ be the characteristic Sturmian sequence of slope $\alpha$. Then

$$
\operatorname{ind}^{*}(\alpha)=1+\operatorname{ice}(\omega) .
$$

Proof. We can easily compute ice $(\omega)$ for such sequences from Corollary 3.5:

$$
\begin{aligned}
\operatorname{ice}(\omega) & =\limsup _{k \rightarrow \infty} \max \left(\frac{\sum_{j=1}^{k+1} a_{j} q_{j-1}}{q_{k}}, 1+\frac{\sum_{j=1}^{k} a_{j} q_{j-1}}{q_{k}}\right) \\
& =\limsup _{k \rightarrow \infty} \frac{\sum_{j=1}^{k+1} a_{j} q_{j-1}}{q_{k}}=\limsup _{k \rightarrow \infty} \frac{q_{k+1}+q_{k}-2}{q_{k}}=\limsup _{k \rightarrow \infty} 1+\frac{q_{k+1}}{q_{k}} \\
& =1+\limsup _{k \rightarrow \infty}\left[a_{k+1} ; a_{k}, \ldots, a_{1}\right]=\operatorname{ind}^{*}(\alpha)-1 .
\end{aligned}
$$

This quantity is finite if and only if the $a_{k}$ are bounded. One has ice $(\omega) \leq 3$ if and only if all but finitely many of the $a_{k}$ are equal to 1 , in which case $\alpha \in \mathbb{Q}(\theta)$ and ice $(\omega)=1+\theta$.

We can recover the shift invariance of ice off the orbit of $\omega$ as follows. Let $\omega(-\alpha)$ be the Sturmian sequence of slope $\alpha$ coding the orbit of $-\alpha$ under $R_{\alpha}$, and let $\omega(1-\alpha)$ be the Sturmian sequence of slope $\alpha$ coding the orbit of $1-\alpha$ under $\widetilde{R}_{\alpha}$. These sequences are the two shift preimages of the characteristic sequence $\omega$, i.e.,

$$
\omega(-\alpha)=0 \omega \text { and } \omega(1-\alpha)=1 \omega .
$$

Since $\sigma\left(0 a_{2} 0 a_{4} \ldots\right)=\sigma\left(a_{1} 0 a_{3} 0 \ldots\right)=0000 \cdots=\Psi(\omega)$, it follows from Proposition 2.8 that

$$
\Psi(\omega(-\alpha))=0 a_{2} 0 a_{4} \ldots, \quad \Psi(\omega(1-\alpha))=a_{1} 0 a_{3} 0 \ldots
$$

Corollary 3.5 shows that for $c \in K_{\alpha}$, ice $\left(\Psi^{-1}(c)\right)$ depends only on the tail of $c$, which is by definition the same as that of $\sigma(c)$ unless $c \in\left\{a_{1} 0 a_{3} 0 \ldots\right.$, $\left.0 a_{2} 0 a_{4} \ldots\right\}$. Thus ice $=$ ice $\circ T$ on $X_{\alpha} \backslash\{\omega(-\alpha), \omega(1-\alpha)\}$.

By Corollary 3.5, one checks that for $\omega(-\alpha), x^{\prime}(2 k) \geq x^{\prime}(2 k+1)$ and $y(2 k) \geq y(2 k+1)$ for all $k$, and

limsup $\max \left(x^{\prime}(2 k), y(2 k)\right)$

$$
k \rightarrow \infty
$$

$$
\begin{aligned}
& =\limsup _{k \rightarrow \infty} \max \left(\frac{\sum_{j=1, \mathrm{odd}}^{2 k+1} a_{j} q_{j-1}}{q_{2 k}}, 1+\frac{\sum_{j=1, \mathrm{odd}}^{2 k} a_{j} q_{j-1}}{q_{2 k-2}}\right) \\
& =\limsup _{k \rightarrow \infty} \max \left(\frac{q_{2 k+1}}{q_{2 k}}, 1+\frac{q_{2 k-1}}{q_{2 k-2}}\right),
\end{aligned}
$$


hence

and similarly

$$
\operatorname{ice}(\omega(-\alpha))=\limsup _{k \rightarrow \infty} \max \left(a_{2 k+1}+\frac{q_{2 k-1}}{q_{2 k}}, 1+a_{2 k-1}+\frac{q_{2 k-3}}{q_{2 k-2}}\right),
$$

$$
\operatorname{ice}(\omega(1-\alpha))=\limsup _{k \rightarrow \infty} \max \left(a_{2 k+2}+\frac{q_{2 k}}{q_{2 k+1}}, 1+a_{2 k}+\frac{q_{2 k-2}}{q_{2 k-1}}\right) .
$$

This implies ice $(\omega(-\alpha)) \leq \operatorname{ice}(\omega)$ and ice $(\omega(1-\alpha)) \leq$ ice $(\omega)$. One may have equality as in the Fibonacci case $(\alpha=\theta=[1 ; 1,1, \ldots])$, as well as a strict inequality as, for instance, for $\alpha=[0 ; 3,1,3,1, \ldots]$.

4.3. The "keep one" sequence. The aim of this section is to prove that there exists a Sturmian sequence of slope $\alpha$ with very little repetition at the beginning, even if $\alpha$ has unbounded partial quotients (and thus $X_{\alpha}$ has arbitrarily large powers in its language).

Proposition 4.1. For every irrational slope $\alpha$ there exists a Sturmian sequence $\omega \in X_{\alpha}$ such that ice $(\omega) \leq 1+\theta$.

Proof. This is a special case of (3) of Proposition 2.1, but we find it interesting to specifically give the $S$-adic expansion of such a point $\omega$. Set $c_{k}=a_{k}-1$ for all $k$ and let $\omega \in X_{\alpha}$ be the corresponding Sturmian sequence. We claim that ice $(\omega) \leq \theta+1$. By Corollary 3.5,

$$
\begin{aligned}
\operatorname{ice}(\omega) & =\limsup _{k \rightarrow \infty} \max \left(\frac{\sum_{j=1}^{k+1} q_{j-1}}{q_{k}}, 1+\frac{\sum_{j=1}^{k} q_{j-1}}{q_{k-1}+q_{k-2}}\right) \\
& =\limsup _{k \rightarrow \infty} \max \left(1+\frac{\sum_{j=1}^{k} q_{j-1}}{q_{k}}, 1+\frac{\sum_{j=1}^{k} q_{j-1}}{q_{k-1}+q_{k-2}}\right) \\
& =1+\limsup _{k \rightarrow \infty} \frac{\sum_{j=1}^{k} q_{j-1}}{q_{k-1}+q_{k-2}} .
\end{aligned}
$$

Our next lemma completes the proof.

LEMma 4.2. The continued fraction convergents $q_{j}$ satisfy

$$
\frac{\sum_{j=1}^{k} q_{j-1}}{q_{k-1}+q_{k-2}}<\theta .
$$

Proof. Our proof is far from elegant and requires consideration of several cases. Let $f_{n}$ be the Fibonacci sequence $f_{0}=0, f_{1}=1$ and $f_{n+1}=f_{n}+f_{n-1}$. Also, set $a_{1}^{\prime}=a_{1}+1$ and $a_{n}^{\prime}=a_{n}$ for $n \geq 2$.

If all of the $a_{j}^{\prime}, j=1, \ldots, k-1$, are equal to 1 then $q_{j}=f_{j+1}$ for $0 \leq j \leq k$ and

$$
\frac{\sum_{j=1}^{k} q_{j-1}}{q_{k-1}+q_{k-2}}=\frac{f_{k+2}-1}{f_{k+1}}<\theta,
$$

since $f_{k+2} / f_{k+1}$ is one of the continued fraction convergents for $\theta$. 
Otherwise we let $l \in\{1,2, \ldots, k-2\}$ be the greatest index for which $a_{l}^{\prime} \neq 1$, or we set $l=1$ if $a_{1}^{\prime}=\cdots=a_{k-2}^{\prime}=1$ (and thus $a_{k-1}^{\prime}>1$ ). We have

$$
q_{r}=f_{r-l+1} q_{l}+f_{r-l} q_{l-1} \quad \text { for } l \leq r \leq k-2,
$$

and from the recursive definitions,

$$
\begin{aligned}
& \frac{\sum_{j=1}^{k} q_{j-1}}{q_{k-1}+q_{k-2}} \\
& =\frac{\left(f_{k-l+2}-1\right) q_{l}+\left(f_{k-l+1}-1\right) q_{l-1}+\left(a_{k-1}^{\prime}-1\right) q_{k-2}+\sum_{j=1}^{l} q_{j-1}}{f_{k-l+1} q_{l}+f_{k-l} q_{l-1}+\left(a_{k-1}^{\prime}-1\right) q_{k-2}} \\
& =\frac{\left(f_{k-l+2}-1+\left(a_{k-1}^{\prime}-1\right) f_{k-l-1}\right) q_{l}+\left(f_{k-l+1}+\left(a_{k-1}^{\prime}-1\right) f_{k-l-2}\right) q_{l-1}+\sum_{j=1}^{l-1} q_{j-1}}{\left(f_{k-l+1}+\left(a_{k-1}^{\prime}-1\right) f_{k-l-1}\right) q_{l}+\left(f_{k-l}+\left(a_{k-1}^{\prime}-1\right) f_{k-l-2}\right) q_{l-1}} \\
& =\frac{\left(f_{k-l+2}+\left(a_{k-1}^{\prime}-1\right) f_{k-l-1}\right) q_{l}+\left(f_{k-l+1}-a_{l}^{\prime}+\left(a_{k-1}^{\prime}-1\right) f_{k-l-2}\right) q_{l-1}+\sum_{j=1}^{l-2} q_{j-1}}{\left(f_{k-l+1}+\left(a_{k-1}^{\prime}-1\right) f_{k-l-1}\right) q_{l}+\left(f_{k-l}+\left(a_{k-1}^{\prime}-1\right) f_{k-l-2}\right) q_{l-1}} \\
& \leq \frac{\left(f_{k-l+2}+\left(a_{k-1}^{\prime}-1\right) f_{k-l-1}\right) q_{l}+\left(f_{k-l+1}-\left(a_{l}^{\prime}-1\right)+\left(a_{k-1}^{\prime}-1\right) f_{k-l-2}\right) q_{l-1}}{\left(f_{k-l+1}+\left(a_{k-1}^{\prime}-1\right) f_{k-l-1}\right) q_{l}+\left(f_{k-l}+\left(a_{k-1}^{\prime}-1\right) f_{k-l-2}\right) q_{l-1}}
\end{aligned}
$$

since $q_{0}+\cdots+q_{l-3}<q_{l-1}$. We shall use the fact that $(a+b) /(c+d)$ is between $a / c$ and $b / d$ for any positive real numbers $a, b, c, d$. have

If $a_{k-1}^{\prime}>1$ then, since $\frac{f_{n+1}+m}{f_{n}+m}<\theta$ for any positive integers $m, n$, we

$$
\frac{f_{k-l+2}+\left(a_{k-1}^{\prime}-1\right) f_{k-l-1}}{f_{k-l+1}+\left(a_{k-1}^{\prime}-1\right) f_{k-l-1}}<\theta, \quad \frac{f_{k-l+1}-\left(a_{l}^{\prime}-1\right)+\left(a_{k-1}^{\prime}-1\right) f_{k-l-2}}{f_{k-l}+\left(a_{k-1}^{\prime}-1\right) f_{k-l-2}}<\theta,
$$

and the desired inequality follows.

We are left to consider the possibility that $a_{k-1}^{\prime}=1$ and $a_{l}^{\prime}>1$. The inequality above simplifies to

$$
\frac{\sum_{j=1}^{k} q_{j-1}}{q_{k-1}+q_{k-2}} \leq \frac{f_{k-l+2} q_{l}+\left(f_{k-l+1}-\left(a_{l}^{\prime}-1\right)\right) q_{l-1}}{f_{k-l+1} q_{l}+f_{k-l} q_{l-1}} .
$$

If $k-l$ is even then

$$
\frac{f_{k-l+2}}{f_{k-l+1}}<\theta, \quad \frac{f_{k-l+1}-\left(a_{l}^{\prime}-1\right)}{f_{k-l}} \leq \frac{f_{k-l+1}-1}{f_{k-l}}<\theta,
$$

and the desired inequality follows. In case $k-l$ is odd, we have $k-l \geq 3$ and $f_{k-l+1} / f_{k-l}<\theta$. Since

$$
\left(a_{l}^{\prime}-1\right) q_{l-1}>\frac{a_{l}^{\prime}-1}{a_{l}^{\prime}+1} q_{l} \geq \frac{1}{3} q_{l}
$$

we have

$$
\frac{\sum_{j=1}^{k} q_{j-1}}{q_{k-1}+q_{k-2}} \leq \frac{\left(f_{k-l+2}-1 / 3\right) q_{l}+f_{k-l+1} q_{l-1}}{f_{k-l+1} q_{l}+f_{k-l} q_{l-1}}
$$


and the observation that $\left(f_{n}-1 / 3\right) / f_{n-1}<\theta$ for $n \geq 5$ completes the proof.

Remarks. By Proposition 3.3, all prefix powers $r \geq 2$ in the "keep one" Sturmian sequence of slope $\alpha$ are of the form

$$
1+\frac{\sum_{j=1}^{k} q_{j-1}}{q_{k}} \text { or } 1+\frac{\sum_{j=1}^{k} q_{j-1}}{q_{k-1}+q_{k-2}} .
$$

We have $q_{k} \geq q_{k-1}+q_{k-2}$ for $k \geq 2$ and thus, by Lemma 4.2 , the Sturmian sequence obtained this way begins in no $1+\theta$ power at all. It is easy to see from the proof of Lemma 4.2 that

$$
\limsup _{k \rightarrow \infty} 1+\frac{\sum_{j=1}^{k} q_{j-1}}{q_{k-1}+q_{k-2}} \leq 1+\theta
$$

with equality if and only if $\left(a_{k}\right)_{k \geq 1}$ has arbitrarily long strings of consecutive ones. Thus,

$$
\text { ice( "keep one") } \leq 1+\theta \text {. }
$$

One can show that equality holds in this last expression if and only if every sequence of slope $\alpha$ has ice $\geq 1+\theta$.

4.4. The Fibonacci case. We prove some characteristic properties of the Fibonacci Sturmian shift $X_{1 / \theta}=X_{\theta-1}$, which we henceforth denote by $X_{\theta}$. Let us recall that according to the results of Section 4.2, the function ice is shift invariant on $X_{\theta}$, and ice $(\omega)=1+\theta$ if $\omega$ belongs to the $\mathbb{Z}$-orbit of the characteristic sequence. The first statement of the following proposition also occurs in [9].

Proposition 4.3. Every $\omega$ in the Fibonacci shift $X_{\theta}$ begins in arbitrarily large cubes except those $\omega$ in the shift orbit of the characteristic sequence. Furthermore, suppose $\omega \in X_{\theta}$ is not in the shift orbit of the characteristic sequence. Then ice $(\omega)=2+\theta$ if and only if the Ostrowski expansion $\left(c_{k}\right)_{k \geq 1}$ of $\omega$ contains arbitrarily long strings of consecutive $0 \mathrm{~s}$.

Proof. Here we have $a_{1}=0$ and $a_{j}=1$ for all $j \geq 2$. Observe that for $\omega \in X_{\theta}$,

$$
\operatorname{ice}(\omega)=\limsup _{k \rightarrow \infty} y(k) \text {. }
$$

Indeed, this is an immediate consequence of the following:

- if $a_{k+1}=c_{k+1}=1$, then $c_{k}=0$, and $y(k)=x^{\prime}(k)+1$;

- if $c_{k+1}=c_{k}=0$, then $y(k)=x^{\prime}(k)$;

- if $c_{k+1}=0$ and $c_{k}=1$, then $c_{k-1}=0$, and $y(k)=1+x^{\prime}(k-2)$.

Let $\omega$ be a Sturmian sequence of slope $\theta$, with Ostrowski expansion $\left(c_{k}\right)_{k \geq 1}$, not belonging to the shift orbit of the characteristic sequence. That is, $\left(c_{k}\right)_{k \geq 1}$ ends with neither $0101010 \ldots$ nor $00000 \ldots$. 
The pattern 001 must appear infinitely often in the sequence $\left(c_{k}\right)_{k \geq 1}$. Consider an integer $k$ for which $\left(c_{k-2}, c_{k-1}, c_{k}\right)=(0,0,1)$. We have

$$
y(k)=1+\frac{q_{k-2}+q_{k-3}+\sum_{j=2}^{k-3}\left(1-c_{j}\right) q_{j-1}}{q_{k-2}} .
$$

One easily proves by induction that for any positive integer $l$,

$$
\sum_{j=2}^{l}\left(1-c_{j}\right) q_{j-1} \geq q_{l-1}-1
$$

with equality if and only if $c_{l-i} \equiv l-i+1 \bmod 2$, for $j=0,1, \ldots, l-1$. Thus, if $k$ is large enough that $\left(c_{j}\right)_{j=1}^{k-3}$ contains two consecutive 0 s, the prefix of $\omega$ of length $q_{k-2}$ has prefix power

$$
y(k) \geq 2+\frac{q_{k-3}+q_{k-4}}{q_{k-2}}=3 .
$$

Consider now an arbitrary index $k$. If $c_{k}=0$ then

$$
y(k)=1+\frac{\sum_{j=2}^{k-1}\left(1-c_{j}\right) q_{j-1}}{q_{k}} \leq 1+\frac{\sum_{j=2}^{k-1} q_{j-1}}{q_{k}}=1+\frac{q_{k+1}-2}{q_{k}}<1+\theta,
$$

and if $c_{k}=1$ then

$$
y(k)=2+\frac{\sum_{j=2}^{k-2}\left(1-c_{j}\right) q_{j-1}}{q_{k-2}}=2+\frac{q_{k-1}-\sum_{j=2}^{k-2} c_{j} q_{j-1}}{q_{k-2}} .
$$

Since $q_{k-1} / q_{k-2} \rightarrow \theta$ as $k \rightarrow \infty$, we see that ice $(\omega)=2+\theta$ if and only if $\left(c_{k}\right)_{k \geq 1}$ contains arbitrarily long strings of consecutive 0 s and infinitely many $1 \mathrm{~s}$.

\section{SMALLEST PREFIX POWERS}

Now we turn our attention to minimizing ice over $X_{\alpha}$ and proving Theorem 1.1, which we recall below.

Theorem 1.1. Let $\alpha=\left[0 ; a_{1}, a_{2}, a_{3}, \ldots\right]$ be an irrational number and $X_{\alpha}$ be the set of all Sturmian sequences of slope $\alpha$. Then there is a Sturmian sequence $\omega \in X_{\alpha}$ with ice $(\omega)=2$ if and only if for each pair of positive integers $(s, t)$ with $s>1$ there are only finitely many $k$ for which $\left(a_{k}, a_{k+1}\right)=$ $(s, t)$ or $\left(a_{k}, a_{k+1}, a_{k+2}\right)=(1,1, t)$.

We deduce from this theorem that if $\min \left(\operatorname{ice}\left(X_{\alpha}\right)\right)=2$ then $\alpha$ has unbounded partial quotients and only finitely many strings of more than two consecutive $1 \mathrm{~s}$ in its sequence of partial quotients $\left(a_{k}\right)_{k \geq 1}$. The set of $\alpha$ with this property has measure zero, since every finite sequence of positive integers appears infinitely many times in the sequence of partial quotients of almost every real number (see for instance [11]). In particular, no Sturmian shift with a quadratic slope can contain a sequence of ice equal to 2 , and 
by Proposition 2.11, there are no substitutive Sturmian sequences $\omega$ with $\operatorname{ice}(\omega)=2$.

5.1. Some first restrictions. Given the partial quotients $a_{k}$ of $\alpha$ we must choose the $c_{k}$ (satisfying the admissibility condition (1)) so as to minimize the lim sup in Corollary 3.5. A couple of observations will help narrow the playing field:

- If $a_{k}-c_{k}>2$ for infinitely many $k$ then ice $(\omega) \geq 3$. Indeed, if $a_{k}-c_{k}$ $\geq 3$, then

$$
x^{\prime}(k-1) \geq \frac{\left(a_{k}-c_{k}\right) q_{k-1}}{q_{k-1}} \geq 3 .
$$

- Given a sequence $c_{k}$ we can define a new sequence $c_{k}^{\prime}$ by setting

$$
c_{k}^{\prime}= \begin{cases}c_{k} & \text { if } a_{k}=c_{k} \text { or } a_{k+1}=c_{k+1}, \\ a_{k}-1 & \text { otherwise. }\end{cases}
$$

The sequence $c_{k}^{\prime}$ also satisfies the admissibility condition (1) and determines a Sturmian sequence of slope $\alpha$, and the only quantities in the formula of Corollary 3.5 which are increased by substituting the $c_{k}^{\prime}$ for the $c_{k}$ are the ones of the form

$$
y(k)=1+\frac{\sum_{j=1}^{k}\left(a_{j}-c_{j}\right) q_{j-1}}{q_{k}-c_{k} q_{k-1}}
$$

where $k$ is an index for which $c_{k}^{\prime} \neq c_{k}$, in which case $c_{k}^{\prime}=a_{k}-1>c_{k}$ and

$$
\begin{aligned}
1+\frac{\sum_{j=1}^{k}\left(a_{j}-c_{j}^{\prime}\right) q_{j-1}}{q_{k}-c_{k}^{\prime} q_{k-1}} & =1+\frac{\sum_{j=1}^{k}\left(a_{j}-c_{j}^{\prime}\right) q_{j-1}}{q_{k-1}+q_{k-2}} \\
& <1+\frac{\sum_{j=1}^{k}\left(a_{j}-c_{j}^{\prime}\right) q_{j-1}}{q_{k-1}} \\
& \leq \frac{\sum_{j=1}^{k}\left(a_{j}-c_{j}\right) q_{j-1}}{q_{k-1}}
\end{aligned}
$$

so that ice of the new sequence is no greater than that of the given sequence.

Consequently, in our quest to minimize ice over $X_{\alpha}$ we need only consider sequences which for each $k$ satisfy

- $c_{k} \in\left\{0, a_{k}-1, a_{k}\right\}$,

- if $c_{k}=0$ or if $c_{k+1}=a_{k+1}$, then $a_{k}=1$.

5.2. Special slopes. We describe those slopes $\alpha$ for which $X_{\alpha}$ has a sequence with ice equal to 2 . First we rule out some of the noncontenders. 
As before,

$$
\begin{aligned}
\alpha & =\left[0 ; a_{1}+1, a_{2}, a_{3}, \ldots\right], \quad x(k)=\mathbf{1}_{a_{k+2}=c_{k+2}}+\frac{\sum_{j=1}^{k+1}\left(a_{j}-c_{j}\right) q_{j-1}}{q_{k}}, \\
x^{\prime}(k) & =\frac{\sum_{j=1}^{k+1}\left(a_{j}-c_{j}\right) q_{j-1}}{q_{k}}, \quad y(k)=1+\frac{\sum_{j=1}^{k}\left(a_{j}-c_{j}\right) q_{j-1}}{q_{k}-c_{k} q_{k-1}} .
\end{aligned}
$$

Proposition 5.1. If $(s, t)$ is a pair of integers with $s>1$ such that $\left(a_{k}, a_{k+1}\right)=(s, t)$ for infinitely many $k$ then every $\omega \in X_{\alpha}$ has

$$
\operatorname{ice}(\omega) \geq 2+\frac{1}{2(s+1)(t+1)+1} .
$$

Proof. From the results of the previous section, we can restrict ourselves to sequences $\left(c_{k}\right)_{k \geq 1}$ which satisfy: for all $k$, if $c_{k}=0$ then $a_{k}=1$. Fix now an index $k$ for which $a_{k}>1$ (and hence $c_{k} \geq 1$ ). There are three cases to consider:

$\mathrm{C} 1: c_{k+2}=a_{k+2}$. Then $c_{k+1}=0$. We have

$$
\begin{aligned}
y(k+2) & =1+\frac{\sum_{j=1}^{k+1}\left(a_{j}-c_{j}\right) q_{j-1}}{q_{k}} \geq 1+a_{k+1}+\frac{a_{k-1} q_{k-2}}{q_{k}} \\
& \geq 1+a_{k+1}+\frac{1}{2 a_{k}+1} .
\end{aligned}
$$

$\mathrm{C} 2: c_{k+2} \leq a_{k+2}-2$. We have

$$
\begin{aligned}
x(k+1) & \geq a_{k+2}-c_{k+2}+\frac{\sum_{j=1}^{k+1}\left(a_{j}-c_{j}\right) q_{j-1}}{q_{k+1}} \\
& \geq a_{k+2}-c_{k+2}+\frac{a_{k} q_{k-1}}{q_{k+1}} \\
& \geq a_{k+2}-c_{k+2}+\frac{1}{2 a_{k+1}+1} .
\end{aligned}
$$

C3: $c_{k+2}=a_{k+2}-1$. Using the fact that $c_{k+1}<a_{k+1}$, which follows here from the hypothesis that $a_{k}>1$, we have

$$
\begin{aligned}
y(k+2) & =1+\frac{q_{k+1}+\sum_{j=1}^{k+1}\left(a_{j}-c_{j}\right) q_{j-1}}{q_{k+1}+q_{k}} \\
& \geq 1+\frac{q_{k+1}+q_{k}+\sum_{j=1}^{k}\left(a_{j}-c_{j}\right) q_{j-1}}{q_{k+1}+q_{k}} \geq 2+\frac{a_{k-1} q_{k-2}}{q_{k+1}+q_{k}} \\
& \geq 2+\frac{1}{2\left(a_{k}+1\right)\left(a_{k+1}+1\right)+1} .
\end{aligned}
$$

In every case, one of $x(k+1), y(k+1)$ and $y(k+2)$ is at least

$$
2+\frac{1}{2\left(a_{k}+1\right)\left(a_{k+1}+1\right)+1} .
$$

The result follows from this fact and Proposition 3.3. 
Proposition 5.2. If $t$ is an integer such that $\left(a_{k}, a_{k+1}, a_{k+2}\right)=(1,1, t)$ for infinitely many $k$ then every $\omega \in X_{\alpha}$ has

$$
\operatorname{ice}(\omega) \geq 2+\frac{1}{8 t+1} .
$$

Proof. Fix an index $k$ for which $a_{k}=a_{k+1}=1$, and set $t=a_{k+2}$. We can save ourselves some labor by noting that in our proof of Proposition 5.1 the assumption $a_{k}>1$ was used only in the third of the three cases, to deduce that $c_{k+1}<a_{k+1}$; in the first two cases the same estimates are valid and we see that one of $x(k+1)$ and $y(k+2)$ is at least $2+1 / 9 \geq 2+1 /\left(8 a_{k+2}+1\right)=$ $2+1 /(8 t+1)$.

In the case that $c_{k+1}=a_{k+1}$ we must have $c_{k+2}<a_{k+2}$; if we replace $k$ with $k+1$ in our proof of Proposition 5.1, we find that one of $x(k+2)$ and $y(k+3)$ is at least

$$
2+\frac{1}{4\left(a_{k+2}+1\right)+1} \geq 2+\frac{1}{8 a_{k+2}+1}=2+\frac{1}{8 t+1} .
$$

5.3. Proof of Theorem 1.1. Finally, we can prove the main theorem.

Proof of Theorem 1.1. One direction follows from the preceding propositions. Let us prove the converse. Let $\alpha$ be as in the statement of the theorem, that is, for each pair of positive integers $(s, t)$ with $s>1$ there are only finitely many indices $k$ for which

$$
\left(a_{k}, a_{k+1}\right)=(s, t) \text { or }\left(a_{k}, a_{k+1}, a_{k+2}\right)=(1,1, t) .
$$

We shall define the sequence $\left(c_{k}\right)_{k \geq 1}$ and check that the Sturmian sequence it represents has ice equal to 2 . Since ice does not depend on the first values of $c_{k}$, we will define the $c_{k}$ for $k$ large enough such that the pattern 111 no longer appears in $a_{k}, a_{k+1}, \ldots$ We just require that the first values of $\left(c_{k}\right)_{k \geq 1}$ satisfy the admissibility condition (1). Here it is:

$$
c_{k}= \begin{cases}a_{k}-1 & \text { if } a_{k}>1, a_{k-1}>1, \\ a_{k}-1 & \text { if } a_{k}>1, a_{k-1}=a_{k-2}=1, \\ a_{k} & \text { if } a_{k}>1, a_{k-1}=1, a_{k-2}>1, \\ 0 & \text { if } a_{k}=1, a_{k-1}>1, \\ a_{k} & \text { if } a_{k}=1, a_{k-1}=1 .\end{cases}
$$

We verify the admissibility condition: If $c_{k}=a_{k}$ then either $a_{k}>1, a_{k-1}=1$ and $a_{k-2}>1$ or $a_{k}=1, a_{k-1}=1$ and thus $a_{k-2}>1$; in both cases we have $c_{k-1}=0$.

Note that $a_{k}-c_{k} \in\{0,1\}$ for all $k \geq 1$, hence $x^{\prime}(k) \leq y(k)$ for every $k \geq 1$. Assume that ice $(\omega)>2$. Then there exists $\varepsilon>0$ such that $y(k) \geq 2+\varepsilon$ holds for infinitely many integers $k$; among those $k$, one of the following four possibilities holds for infinitely many of them: 
$\mathrm{A}: a_{k}>1$ and $c_{k}=a_{k}-1$;

B: $a_{k}>1$ and $c_{k}=a_{k}$;

$\mathrm{C}: a_{k}=a_{k-1}=1$;

$\mathrm{D}: a_{k}=1$ and $a_{k-1}>1$.

CAsE A. Suppose $a_{k}>1$ and $c_{k}=a_{k}-1$. Then either $a_{k-1}>1$ or $a_{k-1}=a_{k-2}=1$. We thus get

therefore

$$
2+\varepsilon \leq y(k) \leq 1+\frac{\sum_{j=1}^{k}\left(a_{j}-c_{j}\right) q_{j-1}}{q_{k-1}+q_{k-2}},
$$

Since

$$
(1+\varepsilon)\left(q_{k-1}+q_{k-2}\right) \leq \sum_{j=1}^{k} q_{j-1} \leq q_{k-1}+q_{k-2}+q_{k-3}+\sum_{j=1}^{k-3} q_{j-1} .
$$

$$
\sum_{j=1}^{k-3} q_{j-1} \leq \sum_{j=1}^{k-3} a_{j} q_{j-1} \leq q_{k-3}+q_{k-4}
$$

we have

$$
\varepsilon\left(q_{k-1}+q_{k-2}\right) \leq 2 q_{k-3}+q_{k-4},
$$

hence

$$
\varepsilon\left(a_{k-1} q_{k-2}+a_{k-2} q_{k-3}\right) \leq 3 q_{k-3} \leq 3 q_{k-2} .
$$

In particular, $\varepsilon\left(a_{k-1} q_{k-2}\right) \leq 3 q_{k-2}$ and $\varepsilon\left(a_{k-2} q_{k-3}\right) \leq 3 q_{k-3}$ hold for infinitely many $k$, therefore there exists a pair of integers $(s, t)$ such that $\left(a_{k-2}, a_{k-1}\right)=(s, t)$ for infinitely many $k$. It follows from our assumption (3) on $\alpha$ that $s=1$. There are two cases to consider:

- $s=t=1$, and thus for infinitely many $k$,

$$
\begin{aligned}
& a_{k}>1, \quad a_{k-1}=a_{k-2}=1, \quad c_{k}=a_{k}-1, \quad c_{k-1}=a_{k-1}, \\
& a_{k-3}>1, \quad c_{k-2}=0, \quad c_{k-3} \geq a_{k-3}-1,
\end{aligned}
$$

and

$$
\begin{aligned}
2+\varepsilon & \leq y(k) \leq 1+\frac{q_{k-1}+q_{k-3}+q_{k-4}+\sum_{j=1}^{k-4}\left(a_{j}-c_{j}\right) q_{j-1}}{q_{k-1}+q_{k-2}} \\
& =1+\frac{q_{k-1}+q_{k-2}+\sum_{j=1}^{k-4}\left(a_{j}-c_{j}\right) q_{j-1}}{q_{k-1}+q_{k-2}},
\end{aligned}
$$

and thus

$$
\varepsilon\left(q_{k-1}+q_{k-2}\right) \leq 2 q_{k-4} .
$$

As

$$
\begin{aligned}
q_{k-1}+q_{k-2} & =q_{k-2}+q_{k-3}+q_{k-3}+q_{k-4}=3 q_{k-3}+2 q_{k-4} \\
& \geq\left(3 a_{k-3}+2\right) q_{k-4}
\end{aligned}
$$

we see that

$$
\varepsilon\left(3 a_{k-3}+2\right) q_{k-4} \leq 2 q_{k-4} .
$$


Since this inequality holds for infinitely many $k$, there exists an integer $s^{\prime}>1$ such that $a_{k-3}=s^{\prime}$, and $a_{k-2}=t=1$ (by (4)) for infinitely many $k$, a contradiction with (3).

- $s=1$ and $t>1$. We thus have $a_{k}>1, a_{k-1}=t>1, a_{k-2}=1$, and $c_{k}=a_{k}-1$. One can assume $a_{k-3}>1$, by assumption (3) on $\alpha$ (the pattern $11 t$ appears only finitely many times). Hence $c_{k-1}=a_{k-1}$ and $c_{k-2}=0$. We thus obtain, by arguing as in case $s=t=1$,

$$
\varepsilon\left((t+2) a_{k-3}+(t+1)\right) q_{k-4} \leq 2 q_{k-4} .
$$

Since this inequality holds for infinitely many $k$, there exists an integer $t^{\prime}$ such that $a_{k-3}=t^{\prime}, a_{k-2}=1$ and $a_{k-1}=t$ for infinitely many $k$, a contradiction with (3).

CASE B. Suppose $a_{k}>1, c_{k}=a_{k}$. Then $a_{k-1}=1, a_{k-2}>1$ and $c_{k-1}=0$. We have

$$
2+\varepsilon \leq y(k)=1+\frac{q_{k-2}+\sum_{j=1}^{k-2}\left(a_{j}-c_{j}\right) q_{j-1}}{q_{k-2}}=2+\frac{\sum_{j=1}^{k-2}\left(a_{j}-c_{j}\right) q_{j-1}}{q_{k-2}},
$$

hence

$$
\varepsilon q_{k-2} \leq q_{k-3}+\sum_{j=1}^{k-3}\left(a_{j}-c_{j}\right) q_{j-1} \leq 3 q_{k-3},
$$

and

$$
\varepsilon a_{k-2} q_{k-3} \leq 3 q_{k-3} .
$$

Since this inequality holds for infinitely many $k$, there exists an integer $s>1$ such that $a_{k-1}=1$ and $a_{k-2}=s$ for infinitely many $k$, a contradiction with (3).

CASE C. Suppose $a_{k}=a_{k-1}=1$. Then, by hypothesis, $a_{k-2}>1, c_{k}=1$ and $c_{k-1}=0$. We have

$$
2+\varepsilon \leq y(k)=1+\frac{q_{k-2}+q_{k-3}+\sum_{j=1}^{k-3}\left(a_{j}-c_{j}\right) q_{j-1}}{q_{k-2}},
$$

that is,

$$
\varepsilon q_{k-2} \leq 3 q_{k-3},
$$

and

$$
\varepsilon a_{k-2} q_{k-3} \leq 3 q_{k-3} .
$$

Since this inequality holds for infinitely many $k$, there once again exists an integer $s>1$ such that $a_{k-1}=1$ and $a_{k-2}=s$ for infinitely many $k$, contrary to hypothesis (3). 
CASE D. Suppose $a_{k}=1$ and $a_{k-1}>1$. Then $c_{k}=0$. One has

$$
\begin{aligned}
2+\varepsilon & \leq y(k) \leq 1+\frac{q_{k-1}+q_{k-2}+\sum_{j=1}^{k-2}\left(a_{j}-c_{j}\right) q_{j-1}}{q_{k}} \\
& =1+\frac{q_{k}+\sum_{j=1}^{k-2}\left(a_{j}-c_{j}\right) q_{j-1}}{q_{k}}
\end{aligned}
$$

hence

$$
\varepsilon q_{k} \leq q_{k-2}+q_{k-3},
$$

and

$$
\varepsilon\left(a_{k-1}+1\right) q_{k-2} \leq \varepsilon\left(q_{k-1}+q_{k-2}\right) \leq 2 q_{k-2} .
$$

These inequalities hold for infinitely many $k$. It follows that for some $s>1$ we have $a_{k}=1$ and $a_{k-1}=s$ for infinitely many $k$, a contradiction with (3).

Acknowledgements. We would like to thank Boris Adamczewski, Jean-Paul Allouche, Fabien Durand and Alain Rémondière for many stimulating discussions.

\section{References}

[1] B. Adamczewski, Répétitions dans les codages de rotations, Adv. Appl. Math. 34 (2005), 1-29.

[2] B. Adamczewski and Y. Bugeaud, On the complexity of algebraic numbers II. Continued fractions, Acta Math., to appear.

[3] J.-P. Allouche, J. P. Davison, M. Queffélec and L. Q. Zamboni, Transcendence of Sturmian or morphic continued fractions, J. Number Theory 91 (2001), 39-66.

[4] P. Arnoux, S. Ferenczi and P. Hubert, Trajectories of rotations, Acta Arith. 87 (1999), 209-217.

[5] P. Arnoux and A. M. Fisher, The scenery flow for geometric structures on the torus: the linear setting, Chinese Ann. Math. Ser. B 22 (2001), 427-470.

[6] P. Arnoux et G. Rauzy, Représentation géométrique de suites de complexité $2 n+1$, Bull. Soc. Math. France 119 (1991), 199-215.

[7] G. Barat and P. Liardet, Dynamical systems originated in the Ostrowski alphaexpansion, Ann. Univ. Sci. Budapest. Sect. Comput. 24 (2004), 133-184.

[8] J. Berstel, Recent results in Sturmian words, in: Developments in Language Theory II, J. Dassow, G. Rozenberg and A. Salomaa (eds.), World Scientific, Singapore, 1996, 13-24.

[9] —, On the index of Sturmian words, in: Jewels are Forever, Springer, Berlin, 1999, 287-294.

[10] V. Berthé, Autour du système de numération d'Ostrowski, Bull. Belg. Math. Soc. Simon Stevin 8 (2001), 209-239.

[11] P. Billingsley, Ergodic Theory and Information, Wiley, New York, 1965.

[12] J. Cassaigne, Special factors of sequences with linear subword complexity, in: Developments in Language Theory II (Magdeburg, 1995), World Scientific, Singapore, 1996, 25-34.

[13] - Limit values of the recurrence quotient in Sturmian sequences, Theoret. Comput. Sci. 218 (1999), 3-12. 
[14] E. M. Coven and G. A. Hedlund, Sequences with minimal block growth, Math. Systems Theory 7 (1973), 138-153.

[15] D. Crisp, W. Moran, A. Pollington and P. Shiue, Substitution invariant cutting sequences, J. Théor. Nombres Bordeaux 5 (1993), 123-137.

[16] D. Damanik, R. Killip and D. Lenz, Uniform spectral properties of one-dimensional quasicrystals, III. $\alpha$-continuity, Comm. Math. Phys. 212 (2000), 191-204.

[17] D. Damanik and D. Lenz, The index of Sturmian sequences, Europ. J. Combin. 23 (2002), 23-29.

[18] I. R. Descombes, Sur la répartition des sommets d'une ligne polygonale régulière non fermée, Ann. Sci. École Norm. Sup. 73 (1956), 283-355.

[19] Y. Dupain and V. T. Sós, On the one-sided boundedness of discrepancy-function of the sequence $\{n \alpha\}$, Acta Arith. 37 (1980), 363-374.

[20] F. Durand, A characterization of substitutive sequences using return words, Discrete Math. 179 (1998), 89-101.

[21] —, Linearly recurrent subshifts have a finite number of non-periodic subshift factors, Ergodic Theory Dynam. Systems 20 (2000), 1061-1078.

[22] - Combinatorial and dynamical study of substitutions around the theorem of Cobham, in: Dynamics and Randomness, Nonlinear Phenom. Complex Systems 7, Kluwer, 2002, 53-94.

[23] S. Ferenczi, Rank and symbolic complexity, Ergodic Theory Dynam. Systems 16 (1996), 663-682.

[24] P. J. Grabner, P. Liardet and R. F. Tichy, Odometers and systems of numeration, Acta Arith. 70 (1995), 103-123.

[25] C. Holton and L. Q. Zamboni, Descendants of primitive substitutions, Theory Comput. Systems 32 (1999), 133-157.

[26] S. Ito and H. Nakada, Approximations of real numbers by the sequence $\{n \alpha\}$ and their metrical theory, Acta Math. Hungar. 52 (1988), 91-100.

[27] J. Justin and G. Pirillo, Fractional powers in Sturmian words, Theoret. Comput. Sci. 255 (2001), 363-376.

[28] J. Lesca, Sur la répartition modulo 1 des suites $(n \alpha)$, Sém. Delange-Pisot-Poitou 8 (1966/67), Théorie des Nombres, Fasc. 1, Exp. 2, Secrétariat Mathématique, Paris, 1966/1967, 9pp.

[29] —, Sur la répartition modulo 1 de la suite no, Acta Arith. 20 (1972), 345-352.

[30] M. Lothaire, Algebraic Combinatorics on Words, Cambridge Univ. Press, Cambridge, 2002.

[31] F. Mignosi, Infinite words with linear subword complexity, Theoret. Comput. Sci. 65 (1989), 221-242.

[32] F. Mignosi and G. Pirillo, Repetitions in the Fibonacci infinite word, RAIRO Inform. Théor. Appl. 26 (1992), 199-204.

[33] F. Mignosi, A. Restivo and S. Salemi, Periodicity and the golden ratio, Theoret. Comput. Sci. 204 (1988), 153-167.

[34] M. Morse and G. A. Hedlund, Symbolic dynamics II: Sturmian trajectories, Amer. J. Math. 62 (1940), 1-42.

[35] A. Ostrowski, Bemerkungen zur Theorie der diophantischen Approximationen, I, II, Abh. Math. Sem. Hamburg 1 (1921), 77-98 and 250-251.

[36] B. Parvaix, Substitution invariant Sturmian bisequences, J. Théor. Nombres Bordeaux 11 (1999), 201-210.

[37] N. Pytheas Fogg, Substitutions in Dynamics, Arithmetics and Combinatorics, V. Berthé, S. Ferenczi, C. Mauduit and A. Siegel (eds.), Lecture Notes in Math. 1794, Springer, 2002. 
[38] R. N. Risley and L. Q. Zamboni, A generalization of Sturmian sequences: Combinatorial structure and transcendence, Acta Arith. 95 (2000), 167-184.

[39] V. T. Sós, On the distribution mod 1 of the sequence n $\alpha$, Ann. Univ. Sci. Budapest. Rolando Eötvös Sect. Math. 1 (1958), 127-134.

[40] M. Stewart, Irregularities of uniform distribution, Acta Math. Acad. Sci. Hungar. 37 (1981), 185-221.

[41] D. Vandeth, Sturmian words and words with a critical exponent, Theoret. Comput. Sci. 242 (2000), 283-300.

[42] L. Vuillon, A characterization of Sturmian words by return words, Europ. J. Combin. 22 (2001), 263-275.

[43] Z.-X. Wen and Z.-Y. Wen, Some properties of the singular words of the Fibonacci word, ibid. 15 (1994), 1-12.

[44] S.-i. Yasutomi, On Sturmian sequences which are invariant under some substitutions, in: Number Theory and Its Applications, S. Kanemitsu and K. Győry (eds.), Kluwer, Dordrecht, 1999, 347-373.

\section{LIRMM}

161 rue Ada

F-34392 Montpellier Cedex 5, France

E-mail: berthe@lirmm.fr

Department of Mathematics

University of North Texas

Denton, TX 76203-5116, U.S.A.

E-mail: luca@unt.edu
Department of Mathematics University of Texas Austin, TX 78712-0257, U.S.A. E-mail: cholton@math.utexas.edu

Received on 20.10.2003

and in revised form on 16.11.2005 